\title{
Cellular Mechanisms Contributing to Response Variability of Cortical Neurons In Vivo
}

\author{
Rony Azouz ${ }^{1}$ and Charles M. Gray² \\ 1 The Center for Neuroscience, ${ }^{2}$ Section of Neurobiology, Physiology, and Behavior, University of California, \\ Davis, California 95616
}

\begin{abstract}
Cortical neurons recorded in vivo exhibit highly variable responses to the repeated presentation of the same stimulus. To further understand the cellular mechanisms underlying this phenomenon, we performed intracellular recordings from neurons in cat striate cortex in vivo and examined the relationships between spontaneous activity and visually evoked responses. Activity was assessed on a trial-by-trial basis by measuring the membrane potential $\left(V_{\mathrm{m}}\right)$ fluctuations and spike activity during brief epochs immediately before and after the onset of an evoked response. We found that the response magnitude, expressed as a change in $V_{\mathrm{m}}$ relative to baseline, was linearly correlated with the preceding spontaneous $V_{\mathrm{m}}$. This correlation was enhanced when the cells were hyperpolarized to reduce the activation of voltage-gated conductances. The output of the cells, expressed as spike counts and latencies, was only moderately correlated with fluctuations in the preceding spontaneous $V_{\mathrm{m}}$. Spike-triggered averaging of $V_{\mathrm{m}}$ revealed that
\end{abstract}

Variation in response strength to repeated presentations of the same stimulus is one of the hallmarks of neuronal activity in sensory systems. In the visual cortex, the response variance to a constant stimulus is often equal to or greater than the mean response (Henry et al., 1973; Tomko and Crapper, 1974; Schiller et al., 1976; Heggelund and Albus, 1978; Rose, 1979; Dean, 1981; Tolhurst et al., 1981, 1983; Scobey and Gabor, 1989; Vogels et al., 1989; Vogels and Orban, 1990; Snowden et al., 1992; Softky and Koch, 1993; Holt et al., 1996). Although small fixational eye movements contribute to response variance in the alert animal (Gur et al., 1997), the fluctuations in neuronal responses remain appreciable. How then are constant sensory features represented by neurons that vary widely in their response strength? A widely held view posits that response variability represents neuronal noise (Burns, 1968; Calvin and Stevens, 1968; Bullock, 1970; Shadlen and Newsome, 1994, 1998). In this framework, reliable signals are conveyed by pooling the activity of many neurons whose individual response variations show no, or perhaps weak, correlation (Britten et al., 1992; Zohary et al., 1994; Shadlen et al., 1996). This mechanism serves to average out the uncorrelated

\footnotetext{
Received Oct. 22, 1998; revised Dec. 22, 1998; accepted Dec. 24, 1998.

This work was supported by grants from the Human Frontiers Science Program (R.A.) and the National Eye Institute (C.M.G). We thank David McCormick for his help in collecting a portion of the data used in this study. We also thank Lee Rognlie-Howes for her excellent care of the animals and technical assistance, and we thank Ad Aertsen for his valuable comments on an earlier version of this manuscript.

Correspondence should be addressed to Dr. Charles Gray, The Center for Neuroscience, 1544 Newton Court, University of California, Davis, CA 95616.

Copyright (C) 1999 Society for Neuroscience 0270-6474/99/192209-15\$05.00/0
}

visually evoked action potentials arise from transient depolarizations having a rise time of $\sim 10 \mathrm{msec}$. Consistent with this, evoked spike count was found to be linearly correlated with the magnitude of $V_{\mathrm{m}}$ fluctuations in the $\gamma(20-70 \mathrm{~Hz})$ frequency band. We also found that the threshold of visually evoked action potentials varied over a range of $\sim 10 \mathrm{mV}$. Examination of simultaneously recorded intracellular and extracellular activity revealed a correlation between $V_{\mathrm{m}}$ depolarization and spike discharges in adjacent cells. Together these results demonstrate that response variability is attributable largely to coherent fluctuations in cortical activity preceding the onset of a stimulus, but also to variations in action potential threshold and the magnitude of high-frequency fluctuations evoked by the stimulus.

Key words: visual cortex; area 17; V1; cat; physiology; cortical dynamics

noise among neuronal responses and yield a precise signal that is conveyed by a population of neurons (Paradiso, 1988; Vogels, 1990; Lee et al., 1998).

A number of studies have demonstrated, however, that neuronal populations in visual cortex exhibit significant covariance in their spontaneous and visually evoked activity (van Kan et al., 1985; Bach and Krueger, 1986; Nelson et al., 1992; Arieli et al., 1995; Nowak et al., 1995). This suggests that response variability arises from nonrandom, correlated interactions in a highly interconnected network and implies that spontaneous activity will have a marked influence on the strength of neuronal responses (Aertsen et al., 1989; Aertsen and Preissl, 1991; Bernander et al., 1991). This prediction was recently confirmed by Arieli et al. (1996), who demonstrated a linear correlation between response strength and the level of spontaneous activity preceding a visual stimulus. These effects are likely to result from the large fluctuations in membrane potential $\left(V_{\mathrm{m}}\right)$ produced by synaptic input onto cortical neurons during spontaneous activity (Pare et al., 1997, 1998).

Despite the broad fluctuations in response to sensory stimuli, cortical neurons are also capable of displaying highly reliable and temporally precise patterns of activity. Neuronal spike trains recorded in vivo can exhibit precisely repeating patterns (Dayhoff and Gerstein, 1983; Frostig et al., 1990; Villa and Abeles, 1990; Abeles et al., 1993; Riehle et al., 1997), engage in synchronous firing with millisecond precision (Singer and Gray, 1995), and follow rapidly varying stimulus inputs with high temporal fidelity [Aertsen et al. (1979); Bair and Koch (1996); Buracas et al. (1998); see also de Ruyter van Steveninck et al. (1997) for an 
example in an invertebrate preparation]. How can such temporal precision occur during broad variations of spontaneous activity?

Recent in vitro studies demonstrate that cortical neurons exhibit temporally precise spike trains in response to broadband membrane currents (Mainen and Sejnowski, 1995; Nowak et al., 1997a). The greatest precision occurs when spikes are driven by rapidly depolarizing currents that are preceded by hyperpolarizing currents (Mainen and Sejnowski, 1995; Nowak et al., 1997a). Events of this form may facilitate the temporal precision of action potentials by transiently reducing the inactivation and rapidly increasing the rate of activation of voltage-gated sodium channels. This enhanced sensitivity to rapid $V_{\mathrm{m}}$ fluctuations suggests two additional sources of variance in the pattern of spike discharges. First, cortical neurons should exhibit variations in action potential threshold in relation to the time course of their $V_{\mathrm{m}}$ fluctuations, and these variations may contribute to spike train variability (Calvin, 1974; Schlue et al., 1974; Schwindt and Crill, 1982; Stafstrom et al., 1984; Heck et al., 1993). Second, an enhanced sensitivity to rapid $V_{\mathrm{m}}$ fluctuations implies that the discharge patterns of cortical neurons should be sensitive to the magnitude of high-frequency fluctuations of $V_{\mathrm{m}}$ resulting from synchronous synaptic inputs (Jagadeesh et al., 1992). This conjecture is supported by two studies demonstrating that response variability is related to variations in synchronous synaptic input (Stevens and Zador, 1998; Zador, 1998).

A further source of response variability is likely to stem from the intrinsic membrane properties of cortical neurons. It is well established that cortical neurons exhibit various discharge patterns in response to constant current inputs (McCormick et al., 1985; Connors and Gutnick, 1990; Gray and McCormick, 1996). The patterns of response are determined by the types, densities, and kinetics of voltage-gated conductances distributed throughout the somatic and dendritic membranes of neurons (Johnston et al., 1996; Yuste and Tank, 1996). Therefore, the $V_{\mathrm{m}}$ of a cell is determined not only by the pattern of its synaptic input, but also by the pattern of current flow through voltage-gated channels that are activated by those inputs. As a result, any variations in $V_{\mathrm{m}}$ that arise from fluctuations in synaptic input are likely to be amplified by the voltage-gated currents intrinsic to the cell.

The current study explores the extent to which response variability can be accounted for by the four mechanisms discussed above: (1) variation in $V_{\mathrm{m}}$ produced by spontaneous synaptic activity, (2) active voltage-gated conductances that give rise to differences in intrinsic membrane properties, (3) variations in the magnitude of stimulus-evoked high-frequency fluctuations of $V_{\mathrm{m}}$, and (4) variations in action potential threshold. We performed intracellular recordings in cat striate cortex in vivo and studied the relation between responses evoked by visual stimuli and the immediately preceding spontaneous activity. Our analysis focused primarily, but not exclusively, on small windows of activity immediately before and after the onset of a response. We looked at the relations between $V_{\mathrm{m}}$ and the rate and latency of action potential discharges, as well as variations in the threshold for action potential generation.

A preliminary report of these findings has been published previously in abstract form (Gray and Azouz, 1997)

\section{MATERIALS AND METHODS}

Adult cats $(2.5-4.0 \mathrm{~kg})$ were initially anesthetized with ketamine (12-15 $\mathrm{mg} / \mathrm{kg})$ and acepromazine $(0.1 \mathrm{mg} / \mathrm{kg})$ and given atropine $(0.05 \mathrm{mg} / \mathrm{kg})$ to reduce salivation. Ringer's solution, containing $2.5 \%$ dextrose, was given intravenously throughout the experiment $\left(4 \mathrm{ml} \cdot \mathrm{kg}^{-1} \cdot \mathrm{hr}^{-1}\right)$. Anesthesia was maintained using halothane $(1.0-1.5 \%)$, in a mixture of nitrous oxide and oxygen (2:1), while the animals were actively ventilated. The electrocardiogram, heart rate (160-200 beats/sec), expiratory $\mathrm{CO}_{2}$ concentration (3.5-4.5\%), and rectal body temperature $\left(37.5-39.0^{\circ} \mathrm{C}\right)$ were monitored throughout the experiment. The animals were mounted in a stereotaxic frame. To minimize pulsations arising from the heartbeat and respiration, the cisterna magna was cannulated, a bilateral pneumothorax was performed, and the animal was suspended by the rib cage to the stereotaxic frame. A craniotomy (3-4 mm diameter) was made overlying the representation of the area centralis of area 17. After the surgery, the animals were paralyzed with pancuronium bromide (Pavulon, $0.3 \mathrm{mg} / \mathrm{kg}$ initial bolus followed by 0.3 $\left.\mathrm{mg} \cdot \mathrm{kg}^{-1} \cdot \mathrm{hr}^{-1}\right)$. The eyes were focused on the screen of a computer monitor at a distance of $57 \mathrm{~cm}$ using corrective, gas-permeable, contact lenses. The nictitating membranes were retracted, and the pupils dilated using ophthalmic Neosynephrine and atropine, respectively. After these procedures, a small opening was made in the dura, and a micropipette was positioned just above the cortical surface. Stability was further improved by application of a $4 \%$ mixture of agar in Ringer's solution to the cortical surface.

Our methods for in vivo intracellular recording have been published previously (Gray and McCormick, 1996). Briefly, intracellular recordings were obtained using $\mathrm{K}^{+}$-acetate-filled $(4 \mathrm{M})$ glass micropipettes beveled to a final resistance of $70-120 \mathrm{M} \Omega$. At the time of recording, the micropipette was positioned immediately above the exposed cortical surface, and the craniotomy was filled with agar-saline. Once respiratory and arterial pulsations had ceased, the pipette was advanced into the cortex using a piezoelectric micromanipulator $(0.5 \mu \mathrm{m}$ resolution). Cell penetrations that yielded stable membrane potentials ranging from -80 to $-55 \mathrm{mV}$, and action potentials of $50-90 \mathrm{mV}$ amplitude, were considered healthy and investigated further. Each cell was given a sequence of hyperpolarizing and depolarizing square wave current pulses (range of -0.5 to $+1.8 \mathrm{nA}$ ) to measure input resistance and assess intrinsic electrophysiological characteristics (McCormick et al., 1985; Larkman and Mason, 1990; Mason and Larkman, 1990; Gray and McCormick, 1996; Kawaguchi and Kubota, 1997). After this protocol, the cell's receptive field (RF) was mapped using mouse-controlled light bars and sine wave gratings. When the recording conditions and cellular responsivity permitted, we evaluated each cell's RF class (simple or complex), ocular dominance, orientation, direction, and spatial frequency selectivity. Once this was completed, we stimulated each cell monocularly through the preferred eye with $20-30$ presentations of a drifting sine wave grating at the preferred orientation, direction, velocity, and spatial frequency. When possible, we also measured the cell's responses to a range of directions and/or spatial frequencies to obtain tuning curves for these parameters. The intracellular signals were stored on a digital tape recorder and digitized off-line at a rate of $20 \mathrm{kHz}$.

In some of the experiments, we performed combined intracellular and extracellular recordings. In these experiments, a tetrode (Gray et al., 1995) or a single extracellular electrode was positioned above the cortical surface immediately adjacent to the intracellular pipette $(100-500 \mu \mathrm{m})$. The cortical surface was sealed with the agar-saline, and the tetrode or electrode was advanced until stable unit activity was isolated. After the $\mathrm{RF}$ properties of the cells were mapped, the intracellular micropipette was then advanced into the cortex until a stable intracellular recording was obtained. The remainder of the protocol was identical to that described above.

The visual stimuli consisted of drifting sine wave gratings (mean luminance of $10-20 \mathrm{~cd} / \mathrm{m}^{2}$ ) that were presented on a dark background and began to move at the onset of their appearance. They were generated by a personal computer and displayed on a 19-inch color monitor $(80 \mathrm{~Hz}$ noninterlaced refresh, $1024 \times 768$ resolution).

Data analysis. The central goal of our analysis was to determine the influence of spontaneous fluctuations of $V_{\mathrm{m}}$ on the magnitude of visually evoked responses (Fig. 1). To achieve this, it was necessary to first identify the onset latency of visual responses so that the transition between spontaneous and visually evoked activity could be defined. Once this was accomplished, we measured the correlation between brief periods of spontaneous and visually evoked activity. Spontaneous activity was assessed by calculating the mean $V_{\mathrm{m}}$ over a window of $64 \mathrm{msec}$ immediately before the response, whereas evoked activity was assessed in two ways. We calculated the mean $V_{\mathrm{m}}$ over a $64 \mathrm{msec}$ window and the total number of spikes over a $128 \mathrm{msec}$ window after the response onset. This enabled us to evaluate the influence of spontaneous fluctuations in $V_{\mathrm{m}}$ on visually evoked fluctuations in both $V_{\mathrm{m}}$ and spike count (Fig. $1 B$ ).

We determined the latency of response onset by visually examining 

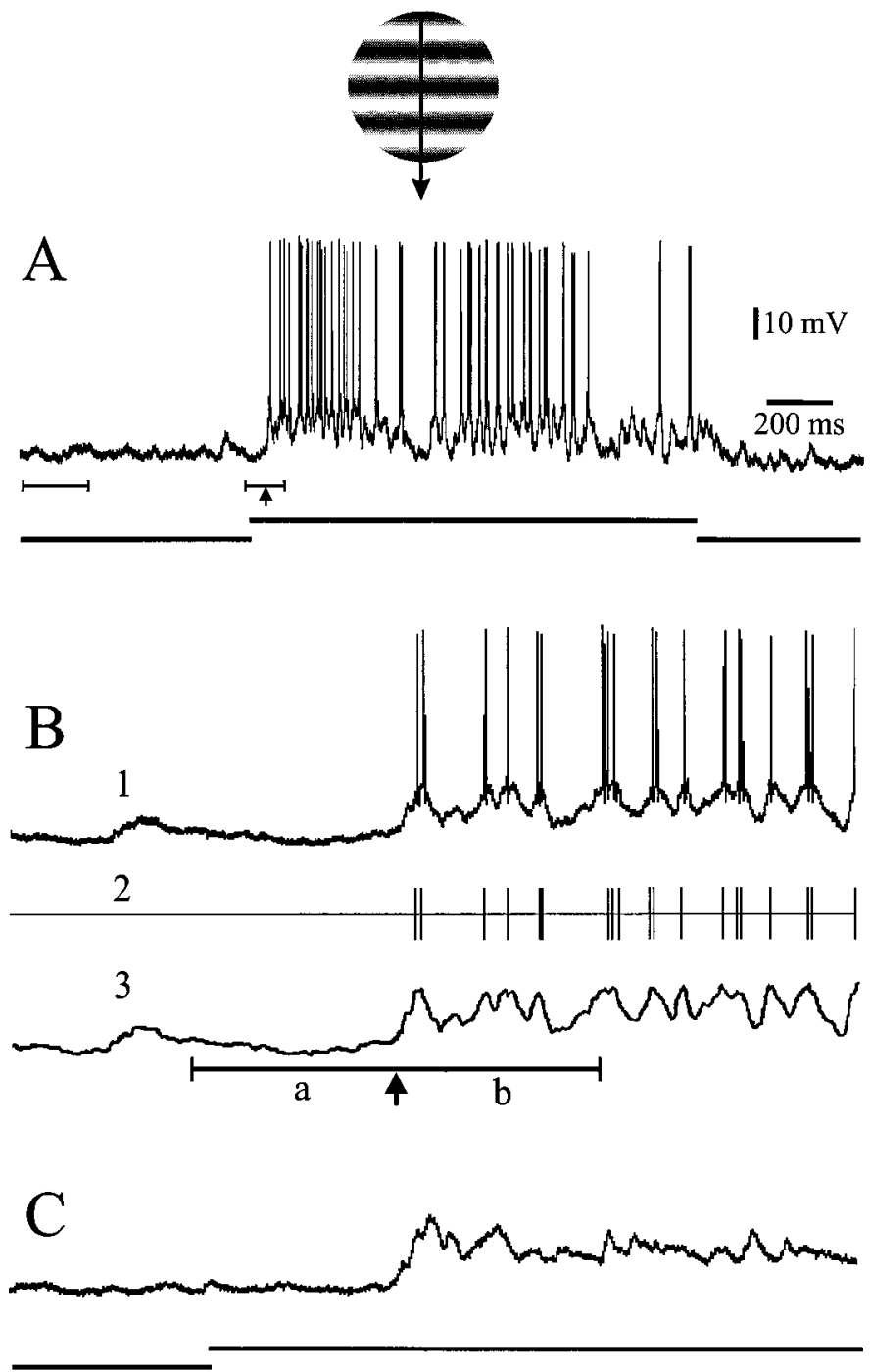

$\overline{20 \mathrm{~ms}}$

Figure 1. This figure illustrates the methodology we used to investigate the relationships between spontaneous and visually evoked activity from cortical neurons recorded intracellularly in vivo. The data are taken from a chattering cell and illustrate the response to a drifting sine wave grating $\left(0.8\right.$ cycles $\left./^{\circ}, 2 \% \mathrm{sec}, 10 \mathrm{~cd} / \mathrm{m}^{2}\right)$ presented to the left eye. $A$, These traces show the membrane potential $\left(V_{\mathrm{m}}\right)$ of the cell (top plot) and the time course of the stimulus (bottom plot) recorded during a single trial. The short horizontal bar on the left indicates the epoch chosen for the baseline $V_{\mathrm{m}}$ measurement, and the one shown during the onset of the stimulus indicates the epochs of activity chosen for analysis. The small arrow marks the onset of the visual response. $B$, Expanded traces of $V_{\mathrm{m}}(1)$, the extracted spike train (2), and the median-filtered $V_{\mathrm{m}}$ (3) illustrating the signals just before and just after the onset of the response (arrow). The bracketed horizontal line marks the boundaries of the adjacent $64 \mathrm{msec}$ windows of spontaneous $(a)$ and visually evoked $(b)$ activity sampled for analysis. When the relation between spontaneous $V_{\mathrm{m}}$ and evoked spike activity was analyzed, the window for the spike analysis was extended to the first $128 \mathrm{msec}$ of the response. $C$, Expanded traces of $V_{\mathrm{m}}$ and the stimulus time course during a single trial while the cell was hyperpolarized below firing threshold. In this and all other measurements, the stimulus began moving at the moment of its appearance.

each trial and subjectively marking the time at which the $V_{\mathrm{m}}$ initiated a clear depolarization (Fig. $1 B$ ). We never allowed this value to be $<35$ msec after the stimulus onset, the mean response latency of LGN Y-cells (Humphrey and Saul, 1992). On most trials, the response onset was easy

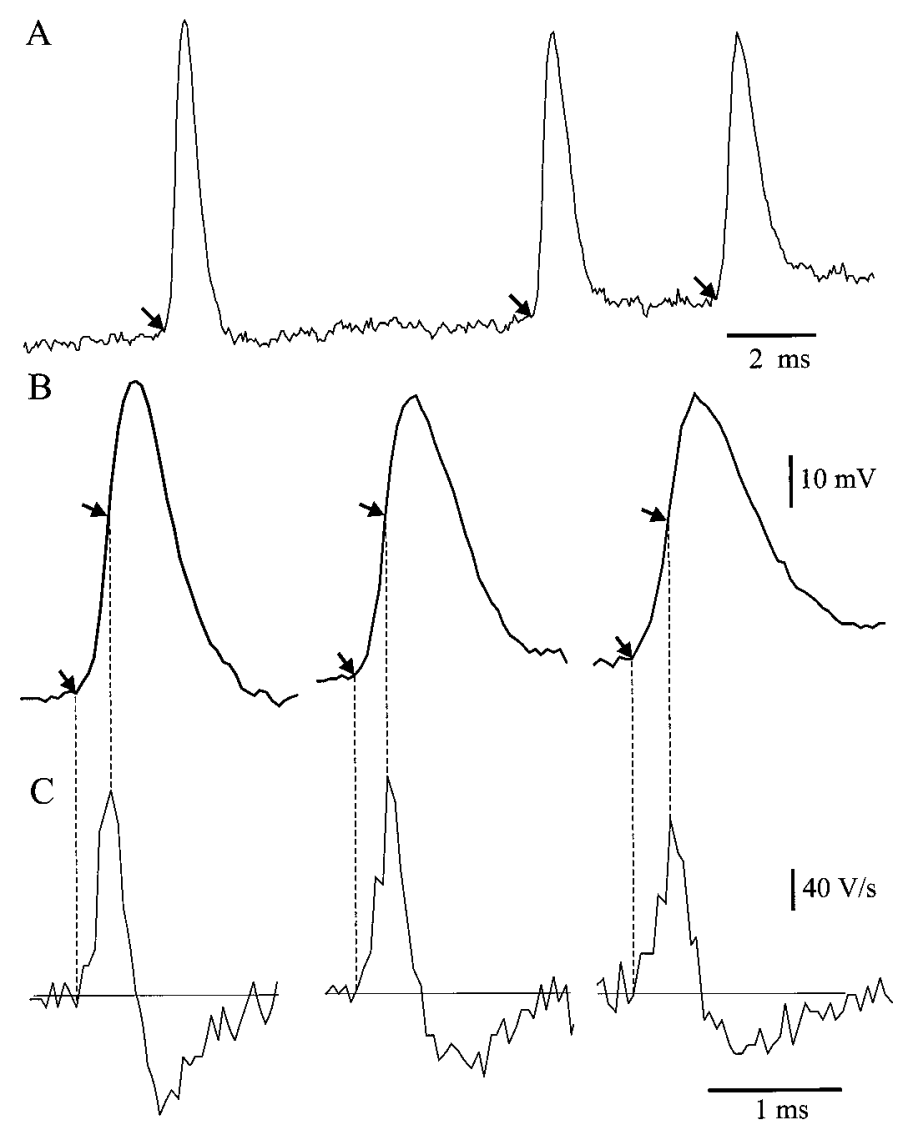

Figure 2. Illustration of the method for calculating action potential threshold. $A$, A brief epoch of data taken from a cell during the response to a visual stimulus. Three action potentials are shown that differ slightly in their threshold voltages (arrows). B, Expanded traces of the three action potentials shown in $A$ illustrating the threshold voltage (lower arrows) and the peak rate of change $\left(d V_{\mathrm{m}} / d t_{\max }\right)$ of the action potential upstroke (upper arrows). $C$, Expanded traces of the rate of change of voltage $\left(d V_{\mathrm{m}} / d t\right)$ for the same three action potentials as shown in $B$. The traces in $B$ and $C$ are plotted in temporal register so that the voltage values and their rates of change can be compared. The pair of vertical dashed lines in each trace illustrates the correspondence between the peak rate of change during the upstroke (right) and the threshold (left). The horizontal lines indicate a value of 0 for $d V_{\mathrm{m}} / d t$.

to identify. On some trials, however, it could be obscured by spontaneous $V_{\mathrm{m}}$ fluctuations (see Fig. $3 A$ ). In these instances, we computed the average $V_{\mathrm{m}}$ across trials and set the response latency to be equivalent to that observed in the average. We did not apply this average measure of response latency to every trial, because fluctuations in latency across trials would have led to erroneous estimates of the transition between spontaneous and evoked activity. Because the earliest component of the response in simple cells (i.e., hyperpolarizing or depolarizing) is dependent on the initial spatial phase of the gratings, we took care to exclude all recordings in which the initial response was hyperpolarizing. This reduced the chances that our correlation measurements may have been comparing two different phases of a visual response.

To evaluate the relationships between the different measures of activity, it was necessary to separate the subthreshold $V_{\mathrm{m}}$ fluctuations from the spike trains. We accomplished this in a multistep sequence. We removed the action potentials from each record by first replacing the data points during a spike with the voltage value recorded at its threshold (see below and Fig. 2). We then resampled the data at $1 \mathrm{kHz}$ and removed any remaining voltage transients by applying a sliding three-point median filter (i.e., the central data point is replaced by the median of the three values). This series of calculations resulted in a continuous record of subthreshold $V_{\mathrm{m}}$ (Fig. $1 B$, trace 3 ). The spike train was stored as a sequence of time stamps at a resolution of $1 \mathrm{msec}$ (Fig. $1 B$, trace 2). 
To make reliable comparisons of $V_{\mathrm{m}}$ across trials, it was necessary to obtain a baseline measure of resting membrane potential. We estimated this value by calculating the mean $V_{\mathrm{m}}$ across trials from the first $250 \mathrm{msec}$ of data collected on each trial (Fig. $1 A$ ). We refer to this value throughout this manuscript as the baseline $V_{\mathrm{m}}$. These epochs occurred well before the period when spontaneous activity was sampled on each trial, and in no instance did the measure of baseline $V_{\mathrm{m}}$ overlap in time with that of the spontaneous $V_{\mathrm{m}}$. The data recorded on each trial were then normalized by subtracting the baseline $V_{\mathrm{m}}$ from each data point. We then assigned a measure of subthreshold activity by calculating the mean $V_{\mathrm{m}}$ of the spontaneous and evoked epochs on each trial. Spike activity was quantified by calculating the number of spikes in the first $128 \mathrm{msec}$ of the response. Spike latency was defined as the time difference between the response onset measured in the $V_{\mathrm{m}}$ and the first spike occurring in the response period.

The relationship between spontaneous and visually evoked activity was examined by calculating the linear correlation coefficient $(r)$ (using the method of least square regression) between all the paired measurements of spontaneous and evoked activity across trials:

$$
r_{\mathrm{xy}}=\frac{\sum_{\mathrm{i}=1}^{\mathrm{n}} x_{\mathrm{i}} y_{\mathrm{i}}}{\sqrt{\left(\sum_{\mathrm{i}=1}^{\mathrm{n}} x_{\mathrm{i}}^{2}\right)\left(\sum_{\mathrm{i}=1}^{\mathrm{n}} y_{\mathrm{i}}^{2}\right)}},
$$

where $x$ and $y$ correspond to any pair of variables (i.e., mean $V_{\mathrm{m}}$, spike count, spike latency), and $n$ is the number of trials. Confidence limits for significant correlation are reported as probability $(p)$ values throughout this manuscript. To control for correlations introduced by the response to the stimulus, we repeated these calculations after randomly shuffling the trial sequence.

The time-dependence of the $V_{\mathrm{m}}$ correlation was further studied by computing the linear correlation between the spontaneous epoch and any other nonadjacent epoch of $V_{\mathrm{m}}$ extending up to $400 \mathrm{msec}$ forward and backward in time. As a control for this measure, we also computed the partial correlation $r_{\mathrm{xy}, \mathrm{z}}(\mathrm{Zar}, 1996)$ on the same epochs of data:

$$
r_{\mathrm{xy}, \mathrm{z}}=\frac{r_{\mathrm{xy}}-r_{\mathrm{xz}} r_{\mathrm{yx}}}{\sqrt{\left(1-r_{\mathrm{xz}}^{2}\right)\left(1-r_{\mathrm{yz}}^{2}\right)}}
$$

where, for example, $x, y$, and $z$ are equal to the mean $V_{\mathrm{m}}$ at times $t, 2 t$, and $3 t$, respectively, and where $t$ is equal to $64 \mathrm{msec}$. This calculation is used to examine the relation between two variables while all other variables involved are kept constant. To put this in terms of our analysis, if $V_{\mathrm{m}}(t)$ is correlated with $V_{\mathrm{m}}(2 t)$ this may influence the correlation between $V_{\mathrm{m}}(t)$ and $V_{\mathrm{m}}(3 t)$. Partial correlation analysis allows us to determine the correlation between $V_{\mathrm{m}}(t)$ and $V_{\mathrm{m}}(3 t)$ while $V_{\mathrm{m}}(2 t)$ is held constant. It thus serves as a useful control for the simple linear correlation measure. To further evaluate the temporal structure of the signals, we also computed the time-lagged $( \pm 100 \mathrm{msec}, 1 \mathrm{msec}$ resolution) autocorrelation function for epochs of spontaneous (500 msec duration) and stimulusevoked (1500-3000 msec duration) activity (Gray et al., 1992). Finally, each of these calculations was repeated on a subset of cells that were hyperpolarized below firing threshold by injecting negative current.

Figure 1 illustrates the application of these methods. The data were collected from a chattering cell (Gray and McCormick, 1996) having a simple receptive field. The top panel depicts the visual stimulus used in these experiments. The plots in $A$ show the activity of the cell in response to a single presentation of the stimulus, and those in $B$ illustrate its decomposition into the corresponding spike train and median-filtered $V_{\mathrm{m}}$. The plots in $C$ illustrate data collected while the cell was hyperpolarized with $0.7 \mathrm{nA}$ of negative current.

We implemented an algorithm to compute the threshold voltage for every action potential in each cell. Our aim was to quantitatively identify the voltage preceding a spike that, once reached, resulted inevitably in the occurrence of an action potential. To achieve this, we computed the maximum rate of change of $V_{\mathrm{m}}\left(d V_{\mathrm{m}} / d t_{\text {max }}\right)$ over three consecutive data points $(150 \mu \mathrm{sec})$ during the upstroke of each action potential (Fig. 2). Using the time point marked by the peak slope (Fig. 2B, upper arrows), we calculated $d V_{\mathrm{m}} / d t$ for each interval of time preceding the peak slope (Fig. $2 C$ ). The spike threshold was determined by measuring the voltage at the onset of each spike at which $d V_{\mathrm{m}} / d t$ first reached an arbitrary fraction of $d V_{\mathrm{m}} / d t_{\max }$ (Fig. $2 B$, lower arrows). This ratio was chosen by assigning a value of $d V_{\mathrm{m}} / d t$ that resulted in a close match to the threshold assigned by careful visual inspection of the raw data at high temporal resolution. For our data set this value was 0.033 . In other words, threshold was defined as the voltage at which the value of $d V_{\mathrm{m}} / d t$ preceding a spike first became $\leq 1 / 30$ of $d V_{\mathrm{m}} / d t_{\max }$. For some cells $(n=5)$ it was difficult to obtain a close match between the objective and subjective assignments of spike threshold. To avoid ambiguity in the results, we discarded these cells from the threshold analysis.

We further considered two possible sources of error in our measurements of action potential threshold. First, while computing the range of spike thresholds for each cell, it became apparent that slow drifts in membrane potential could have a major impact on this measure. To eliminate the uncontrolled variance in spike threshold resulting from these slow drifts in $V_{\mathrm{m}}$, we computed the mean spike threshold for each trial and subtracted this value from each individual threshold value measured on the same trial. This enabled us to pool all the threshold values across trials to determine the range of variation of this parameter. However, this normalization prevents us from measuring the absolute value of threshold voltage and also prevents us from detecting slow changes in threshold over time, as might occur during visual adaptation.

Second, we considered the additional possibility that unspecified instrumentation errors or cellular deterioration could contribute to unusually large fluctuations in action potential threshold. This could occur, for example, if the recorded voltages were fluctuating because of some nonphysiological influence. In this case, the membrane potential fluctuations would be superimposed on voltage fluctuations attributable to some uncontrolled source. To control for this possibility, we computed the SD of peak action potential voltages for each cell to identify any cells in our sample that exhibited unusually large voltage fluctuations. We reasoned that nonphysiological voltage fluctuations would lead to a range of action potential amplitudes that is substantially larger than would be expected from a healthy cell in the absence of instrumentation error. Using this procedure, we identified one cell that displayed fluctuations in spike amplitude that were much greater than the remaining 46 cells. We therefore excluded this cell from all of the analyses in this study.

\section{RESULTS}

The results of this study are taken from 52 neurons that were selected from a larger sample of over 150 intracellular recordings. The cells had stable membrane potentials for durations of 20-90 min and were visually stimulated with drifting sine wave or square-wave gratings. These stimuli enabled us to detect the abrupt onset of response and to clearly distinguish evoked responses from the immediately preceding spontaneous activity.

\section{The relationship of evoked $\boldsymbol{V}_{\mathrm{m}}$ to spontaneous $\boldsymbol{V}_{\mathrm{m}}$}

For each of the cells in our sample $(n=52)$, we assessed the relationship between $V_{\mathrm{m}}$ preceding and immediately after the onset of visually evoked responses. As illustrated in Figure 3, the magnitude of the evoked response was highly correlated with the preceding spontaneous activity. The evoked response was largest when the preceding $V_{\mathrm{m}}$ was depolarized and reduced in amplitude when it was more hyperpolarized (Fig. 3A,B). This relationship was well approximated by a linear function (Fig. $3 D$ ), as indicated by the high linear correlation coefficient $(r=0.91, p$ $\ll 0.0001 ; n=40$ trials). We found a similar significant correlation for $85 \%$ of the cells in the sample $\left(r_{\text {mean }}=0.78 \pm 0.12\right)$. This correlation was not restricted, however, to the relation between spontaneous activity and the evoked response, but held for contiguous epochs of spontaneous activity as well (Fig. 3C) $(r=0.94$, $p \ll 0.0001 ; n=40$ trials). As before, this significant correlation was found for most of the cells in the sample $\left(90 \%, r_{\text {mean }}=0.83 \pm\right.$ 0.11). Interestingly, the correlation between adjacent epochs of spontaneous $V_{\mathrm{m}}$ was significantly greater than that occurring between adjacent epochs of spontaneous and evoked $V_{\mathrm{m}}(p \ll$ $0.001)$. Finally, to control for correlations induced by the stimulus presentation, we repeated the calculations after randomly shuffling the trial sequence. None of the cells in our sample displayed 


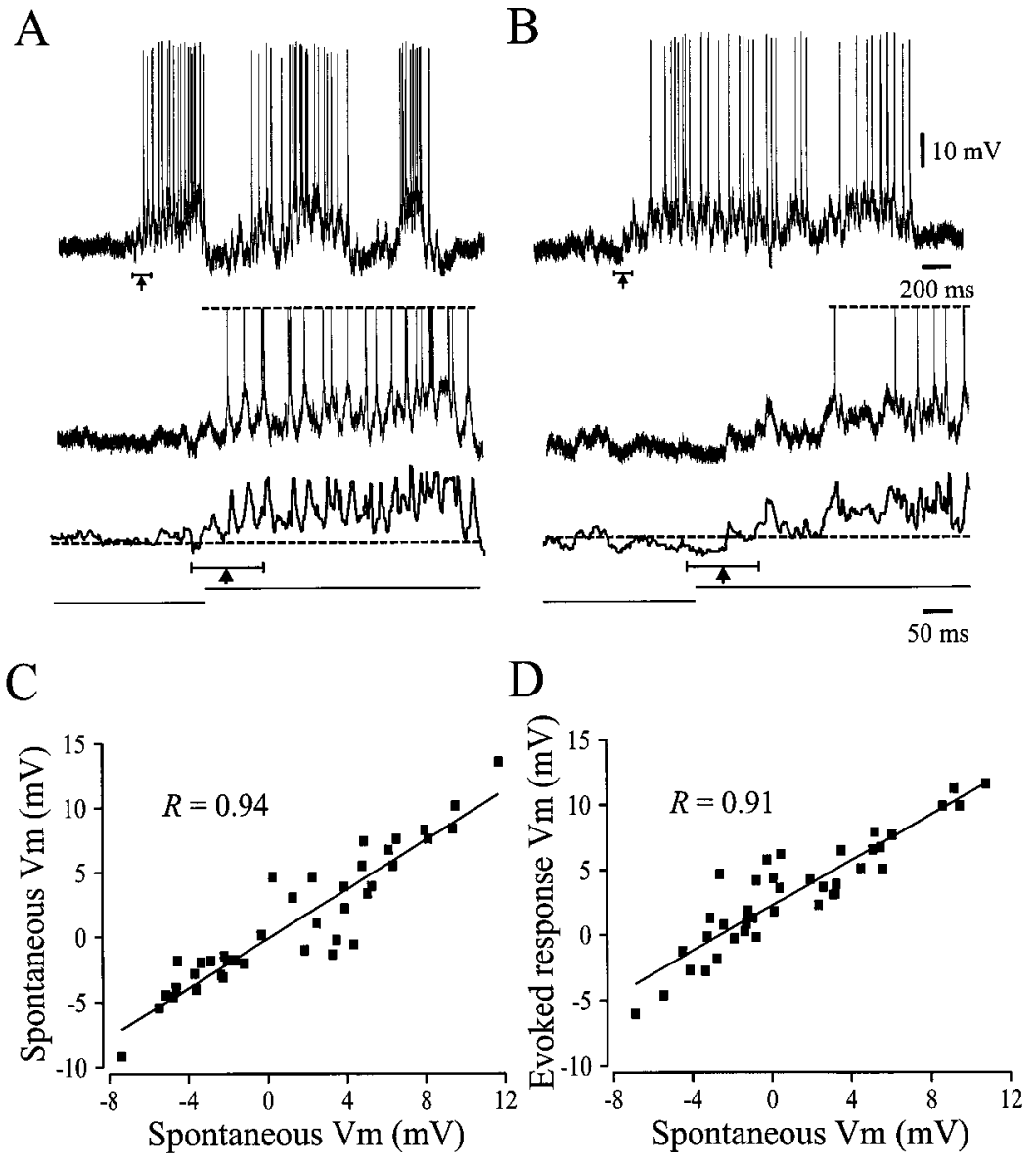

Figure 3. Fluctuations in spontaneous and evoked $V_{\mathrm{m}}$ are linearly correlated. $A, B$, Raw data collected on two separate trials from the same cell as shown in Figure 1. The top plots display the entire duration of each of the two trials. The small arrows mark the onset of the visual response, and the bracketed horizontal line indicates the epochs chosen for the correlation analysis. The middle plot shows the raw data at an expanded time scale. The action potentials have been truncated as indicated by the dashed lines. The bottom plots show the median-filtered data for the same epochs. The dotted line indicates the baseline membrane potential, the bracketed lines indicate the epochs chosen for analysis, and the bottom horizontal line indicates the stimulus time course. The visual response latencies are marked by the arrows. Note that the evoked activity was enhanced when the spontaneous $V_{\mathrm{m}}$ was depolarized (bottom trace in $A$ ) and reduced when the spontaneous $V_{\mathrm{m}}$ was hyperpolarized (bottom trace in $B$ ). $C$, Scatter plot of the mean $V_{\mathrm{m}}$ computed from two adjacent $64 \mathrm{msec}$ epochs of spontaneous activity preceding the visual response on each trial. $D$, Scatter plot of the mean $V_{\mathrm{m}}$ of the evoked response and its immediately preceding spontaneous activity on each trial. In $C$ and $D$ the straight line shows the linear regression fit to the data. a significant correlation after the shuffling procedure, demonstrating that the correlated fluctuations in $V_{\mathrm{m}}$ are independent across trials.

We further studied the time course of these correlations by calculating the linear correlation between the spontaneous $V_{\mathrm{m}}$ preceding the response and epochs of the same duration (64 msec) located at multiples of 64 msec backward and forward in time. This calculation revealed that the magnitude of significant correlation (Fig. $4 A$ ) and the percentage of cells displaying significant correlation (Fig. $4 B$ ) decreased with temporal delay, the rate of decay being greater for the visually evoked activity. We considered the possibility that the correlations between nonadjacent epochs could be explained by the high correlation of $V_{\mathrm{m}}$ between adjacent epochs. To test this notion, we performed two calculations. Using the same epochs of data, we computed the partial correlation of $V_{\mathrm{m}}$ as a function of temporal delay (Fig. $4 C$ ) (see Materials and Methods). This calculation revealed that the correlation between nonadjacent epochs was caused by the high correlation between adjacent epochs. To further evaluate the time course of this correlation, we computed the autocorrelation function of $V_{\mathrm{m}}$ at a resolution of $1 \mathrm{msec}$ on separate epochs sampled during spontaneous and visually evoked activity. We found that the autocorrelation of $V_{\mathrm{m}}$ computed for each cell decayed rapidly and could be fit by a simple exponential function with a single decay time constant (mean $\tau_{\text {spontaneous }}=38 \pm 26$ msec; mean $\tau_{\text {stimulus }}=37 \pm 30 \mathrm{msec}$ ) (Fig. $4 D$ ). Together these results demonstrate that the persistent correlations measured at a low temporal resolution (Fig. 4A,B) are not the result of lowfrequency fluctuations in $V_{\mathrm{m}}$, but rather result from the high correlation between adjacent epochs.

\section{Contribution of intrinsic membrane properties}

Because the stimuli used in these experiments led to vigorous suprathreshold responses, we reasoned that the activation of voltage-gated conductances in the cells might contribute to the correlation between spontaneous and visually evoked activity. To estimate the influence of these intrinsic mechanisms, we compared the correlations between spontaneous and evoked $V_{\mathrm{m}}$ in a subset of the cells $(n=14)$ under control conditions with those occurring when the cells were hyperpolarized to prevent them from reaching threshold. An example of the results of these measurements is shown in Figure $5 A$. In this and all other cells tested, we found that hyperpolarization with steady current injection resulted in a general enhancement of $V_{\mathrm{m}}$ correlation (Fig. $5 B)$. However, this influence was not apparent between the early component of the evoked response and the immediately preceding spontaneous $V_{\mathrm{m}}$ (resting $V_{\mathrm{m}}$ : mean $r=0.72 \pm 0.21$; hyperpolarized $V_{\mathrm{m}}$ : mean $r=0.76 \pm 0.24$ ), suggesting that the early correlation is dominated by the synaptic response to the stimulus. These findings indicate that activation of voltage-gated conductances makes a significant contribution to the variability of spontaneous and evoked activity. However, because hyperpolarization will also change the amplitude of synaptically evoked $V_{\mathrm{m}}$ fluctuations, this effect may also have contributed to the observed changes in correlation.

\section{The relationship between spontaneous $V_{m}$ and evoked discharges}

It is well established that cortical neurons display significant variability in the latency and number of spikes that occur in response to repeated presentation of an identical visual stimulus. 
A

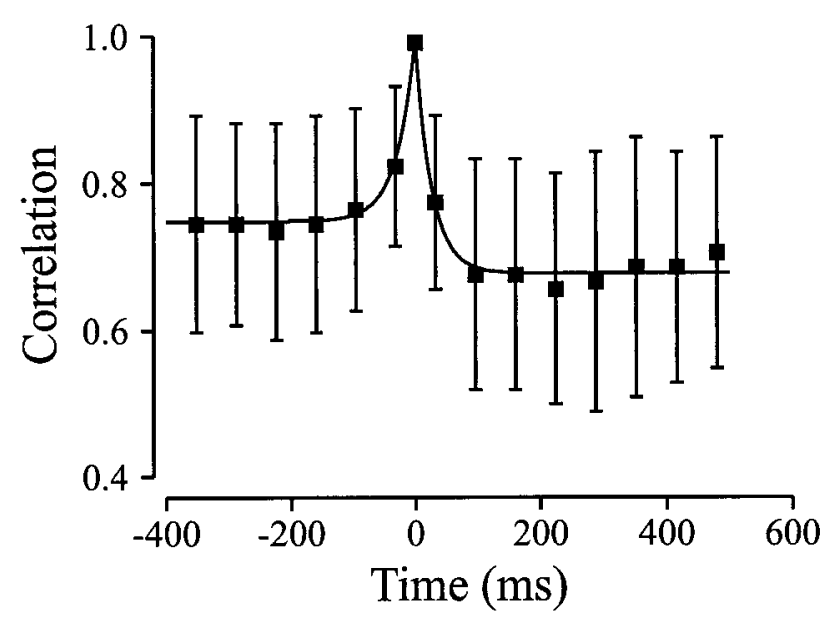

B

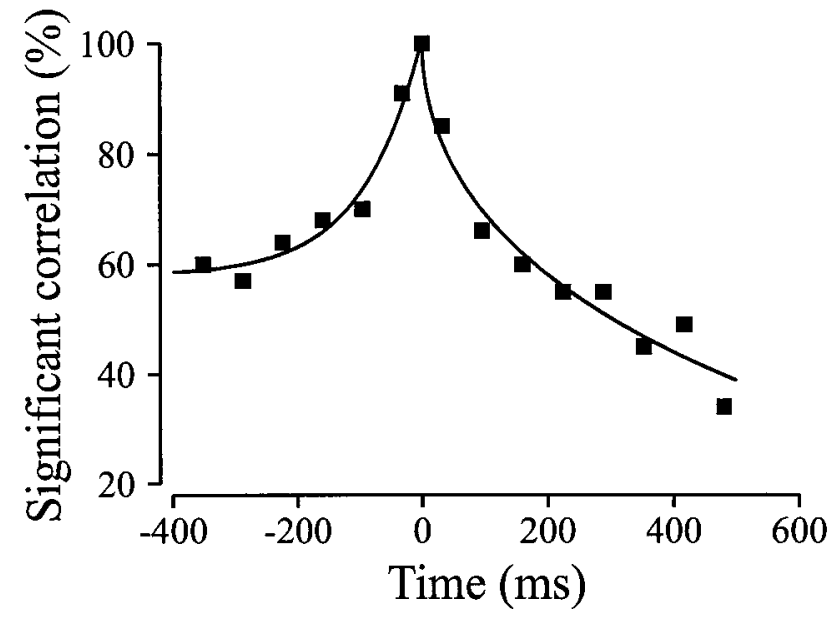

$\mathcal{C}$

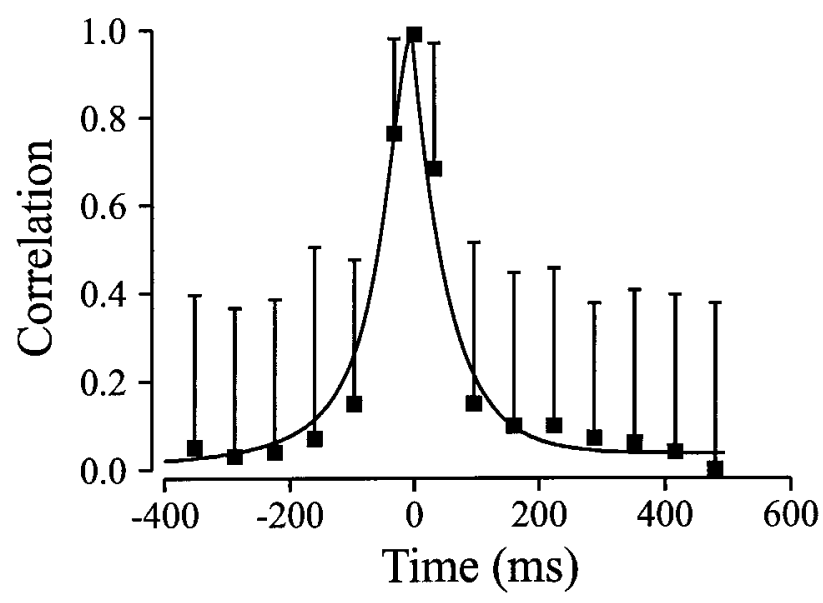

$\mathrm{D}$

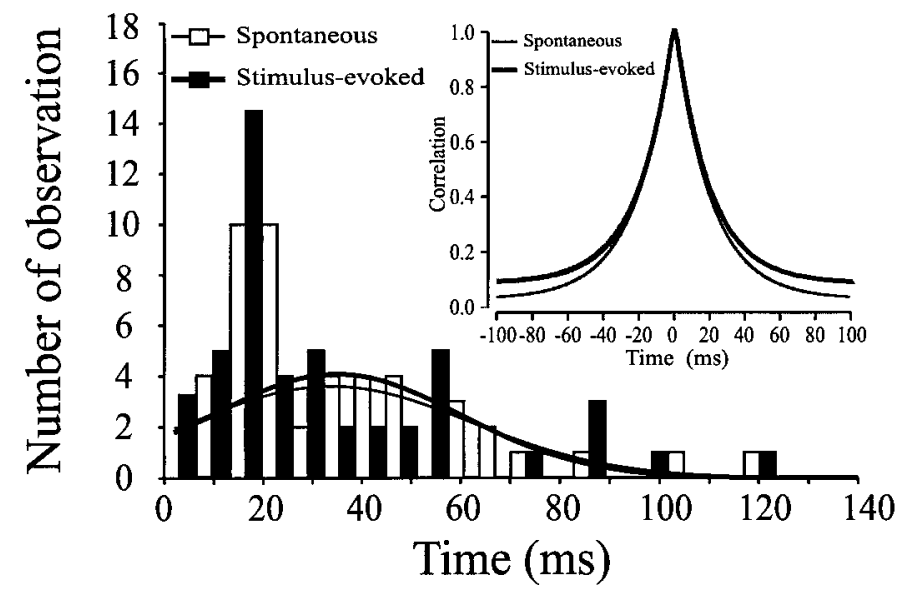

Figure 4. Time course of membrane potential correlation. $A$, The mean and SD of the correlation coefficient as a function of time lag for all the cells having a significant linear correlation. The value at time 0 displays the autocorrelation computed from the spontaneous $V_{\mathrm{m}}$ immediately preceding the response. Points lying to the left and right display the correlation between this epoch and the preceding spontaneous and evoked activity, respectively. Each point in the plot is computed from a different fraction of the cells. $B$, The percentage of cells at each time lag showing a significant linear correlation. $C$, The mean and SD of the partial correlation coefficient as a function of time lag for all the cells in the sample. Note the rapid fall-off in correlation magnitude. $D$, Histogram of the decay time constant computed from the autocorrelation function from the entire sample of cells. The unfilled and filled bars show the values obtained from the spontaneous and stimulus-evoked data, respectively. The inset shows the autocorrelation function computed from the spontaneous (thin line) and stimulus-evoked (thick line) activity in one cell. The autocorrelation functions and the data displayed in $A$ and $C$ were fit by the equation $y=a+b e^{-\mathrm{x} / \mathrm{c}}$. The data in $B$ were fit by the equation $y=a+b x^{\mathrm{c}}$.

Here, we sought to determine the relation between this variability and the underlying $V_{\mathrm{m}}$ fluctuations preceding the response. On each trial, we calculated the latency of the first spike after the response onset and the number of spikes occurring in the first 128 msec of the response and compared these data with the mean $V_{\mathrm{m}}$ occurring during the $64 \mathrm{msec}$ period preceding the response. An example of the results of this analysis is illustrated in Figure 6 and is taken from the same cell as shown in Figures 1 and 3. The cell showed considerable response variability. The number of spikes in the first $128 \mathrm{msec}$ after response onset ranged from 3 to 21, and the latency ranged from 1 to $320 \mathrm{msec}$. This variation in the latency and magnitude of the response was weakly correlated with the preceding $V_{\mathrm{m}}$. Although the number of spikes increased and the latency to the first spike decreased in proportion to the $V_{\mathrm{m}}$ preceding the response onset (Fig. $6 C, D$ ), the strength of the correlation was much lower than that observed for $V_{\mathrm{m}}$ alone. This result also held for the entire sample of cells. A significant correlation between spontaneous $V_{\mathrm{m}}$ and spike count occurred for $51 \%$ of the cells, and the mean value of this correlation was $0.56 \pm 0.15$. Similarly, spike latency was significantly correlated with spontaneous $V_{\mathrm{m}}$ in $30.6 \%$ of the cells, and the mean value was $0.61 \pm 0.18$.

Examination of the time course of correlation for the whole population of cells further revealed that spontaneous $V_{\mathrm{m}}$ and evoked spike count were weakly correlated over time (Fig. 7). The mean correlation decreased from an initial value of 0.39 to a value of 0.21 in $\sim 500 \mathrm{msec}$. As with the previous observations of $V_{\mathrm{m}}$, the partial correlation analysis revealed that the correlations occurring over long temporal delays could be explained by the high correlations between adjacent epochs. 
A
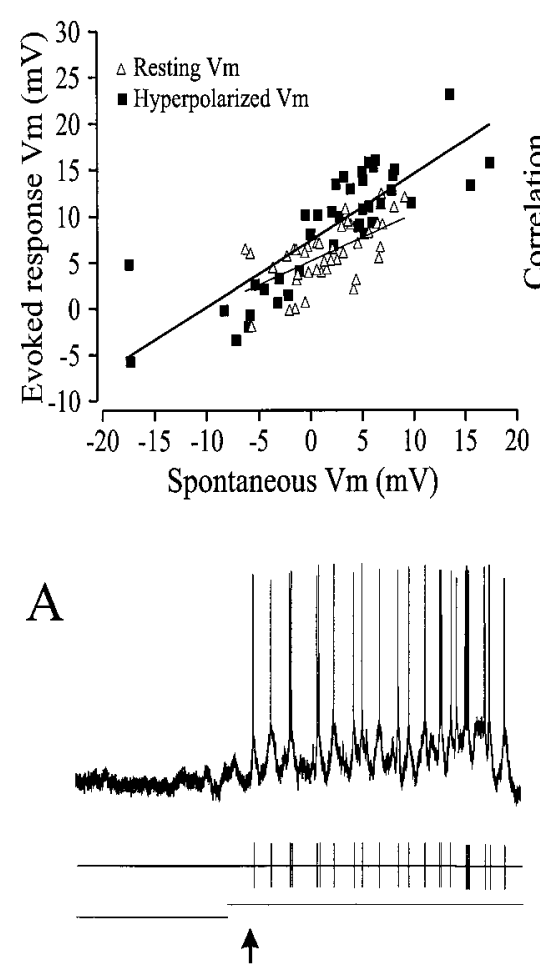

$\mathrm{C}$

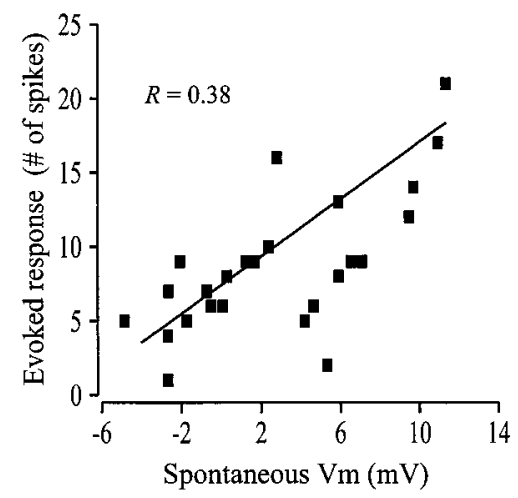

B

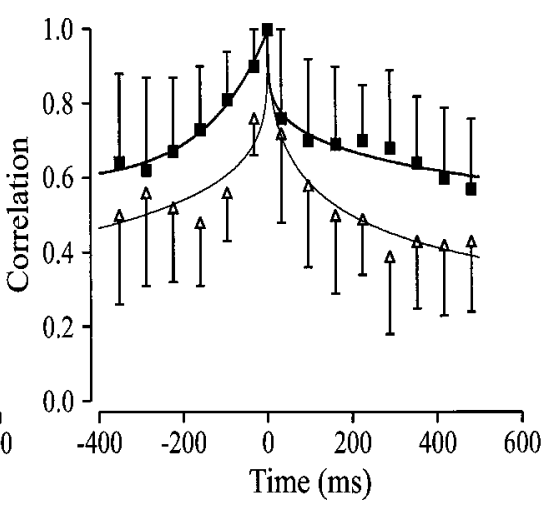

Figure 5. Correlation magnitude is increased by membrane hyperpolarization. $A$, Scatter plots of the spontaneous versus visually evoked $V_{\mathrm{m}}$ recorded across trials under control conditions $(\triangle)$ and when the cell was hyperpolarized with -0.8 $\mathrm{nA}$ of current (ם). The thin and thick lines depict the linear regression fit to the data collected under control $(r=0.76)$ and hyperpolarized $(r=0.81)$ conditions, respectively. $B$, Mean and SD of the linear correlation coefficient for the same cell shown in $A$ as a function of time lag for the control $(\triangle)$ and hyperpolarized ( $\square$ ) conditions, respectively. The filled square at time 0 displays the autocorrelation for both conditions. Note that the mean level of correlation at all time lags is higher when the cell is hyperpolarized below firing threshold. The data were fit by the equation $y=a+b x^{\mathrm{c}}$.

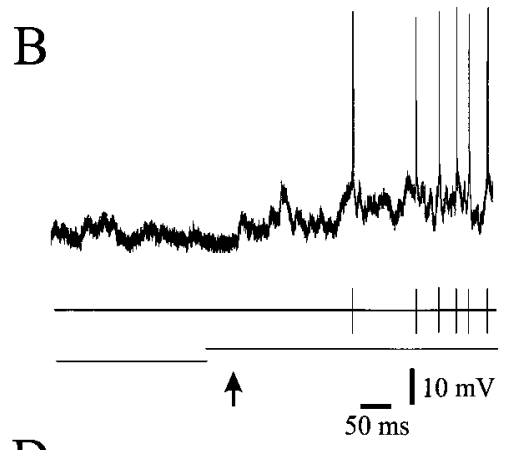

$\mathrm{D}$

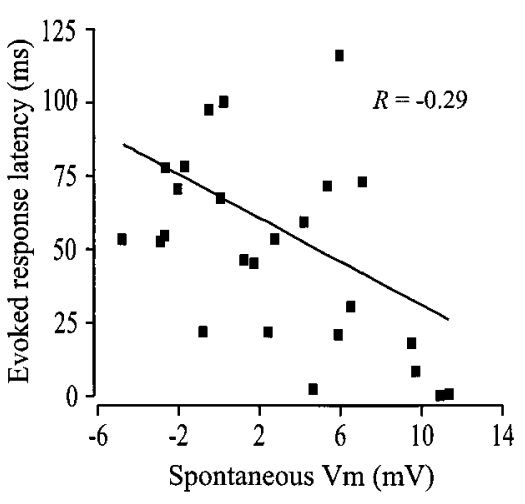

Figure 6. The latency and number of visually evoked spikes are linearly correlated with the spontaneous $V_{\mathrm{m}}$ immediately preceding the visual response. $A, B$, Raw data (top plots) and corresponding spike trains (bottom plots) for the same two trials displayed in Figure $3 A$. The arrows mark the response latency as determined from the change in $V_{\mathrm{m}}$. $C$, Scatter plot of the number of spikes occurring in the first $128 \mathrm{msec}$ of the response versus the mean spontaneous $V_{\mathrm{m}}$ in the $64 \mathrm{msec}$ preceding the response onset. $D$, A similar scatter plot between latency to the first spike and the spontaneous $V_{\mathrm{m}}$. Note that spike count increases and the latency of the first spike decreases in proportion to the spontaneous $V_{\mathrm{m}}$.

In general, these relatively weak correlations indicate that the variability in response discharge cannot be accounted for solely on the basis of spontaneous fluctuations of $V_{\mathrm{m}}$. There must be additional variance introduced during the response to the stimulus that leads to fluctuations in spike initiation. We examined this issue by calculating the correlation of evoked $V_{\mathrm{m}}$ with the number and latency of evoked spikes in a $128 \mathrm{msec}$ epoch after response onset. Examples of these relationships are shown for a single cell in Figure 8, demonstrating that both the number of evoked spikes and the latency to the first spike are loosely correlated to the mean $V_{\mathrm{m}}$ during the response. These results were typical of those observed across the entire sample of cells. Evoked spike count was significantly correlated with the evoked $V_{\mathrm{m}}$ in $57 \%$ of the cells, and the mean correlation coefficient of this group of cells was relatively low (mean $r=0.45 \pm 0.18$ ). Analysis of response latencies revealed that the onset of spike discharge was also weakly correlated to the mean $V_{\mathrm{m}}$ evoked by the stimulus. Only $20 \%$ of the cells in our sample displayed a significant correla- tion between evoked $V_{\mathrm{m}}$ and latency to the first spike of the response. Among this group, the mean correlation coefficient was $0.69 \pm 0.21$.

These results may appear counterintuitive at first glance. However, they may be consistent with the notion that action potentials arise from brief depolarizations reflecting synchronous synaptic input (Nowak et al., 1997b). To test this notion, we performed two calculations on the evoked responses recorded in our cell population. First, we computed the spike-triggered average (STA) of the membrane potential (Komatsu et al., 1988; Jagadeesh et al., 1992; Mainen and Sejnowski, 1995; Nowak et al., 1997a) to determine the average time course of the depolarization associated with spike discharge. This was done separately for the spontaneous and visually evoked activity for cells that displayed a sufficient number of spontaneously occurring spikes. We quantified the peaks in the STAs by measuring their widths and rise times (Fig. 9). These calculations confirmed previous reports (Mainen and Sejnowski, 1995; Nowak et al., 1997a,b) that action 


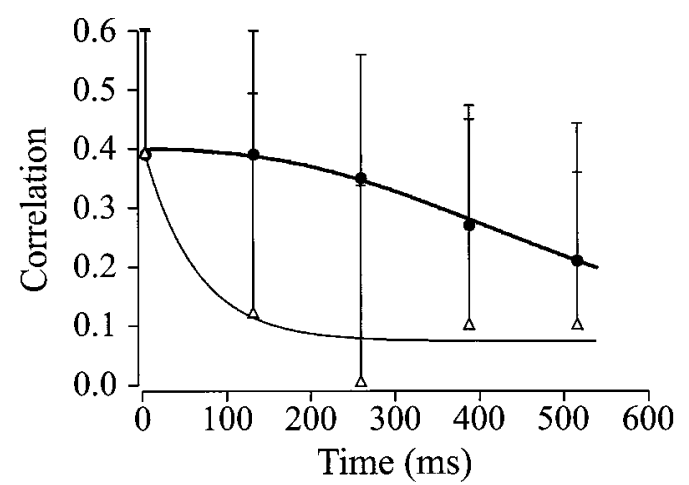

Figure 7. Mean and SD of the correlation between evoked spike count and the preceding $V_{\mathrm{m}}$ as a function of time lag $(n=52)$. The filled circles depict the linear correlation coefficients and are fit by the equation $y=$ $1 /\left(a+b x^{3}\right)$, and the open triangles depict the partial correlation coefficients and are fit by the equation $y=a+b e^{-\mathrm{x} / \mathrm{c}}$. Note that the mean correlation between spontaneous $V_{\mathrm{m}}$ and evoked spike count is significantly lower than the correlation between spontaneous and evoked $V_{\mathrm{m}}$. As with the $V_{\mathrm{m}}$ correlation depicted in Figure 4, the partial correlation decays much more rapidly with time lag.

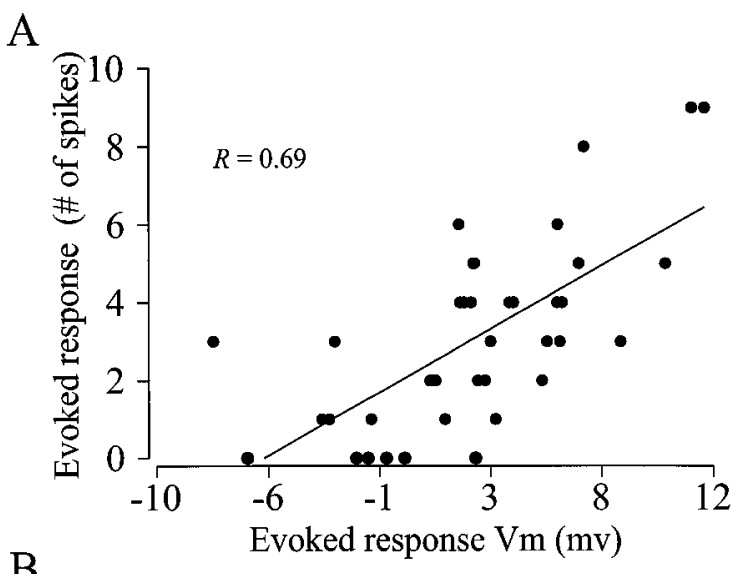

$\mathrm{B}$

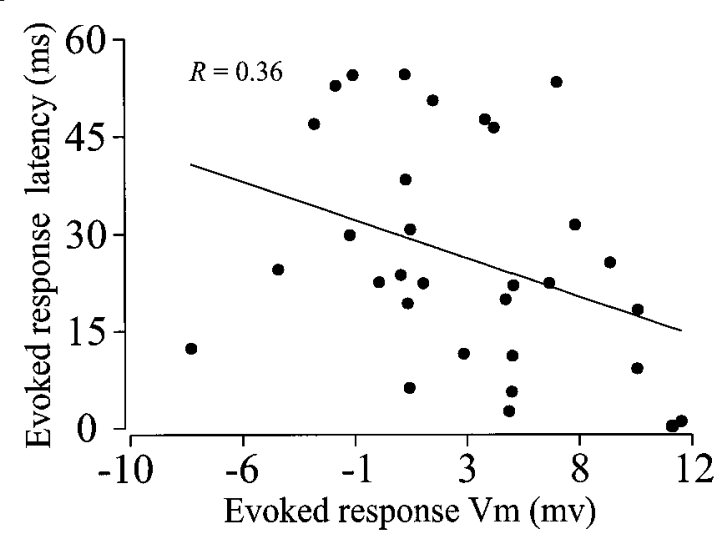

Figure 8. Visually evoked changes in $V_{\mathrm{m}}$ are moderately correlated with the latency and number of evoked spikes. $A$, Scatter plot of the number of visually evoked spikes versus the mean evoked $V_{\mathrm{m}}$ in the $128 \mathrm{msec}$ after the response onset. $B$, Similar scatter plot between latency to the first spike and the mean evoked $V_{\mathrm{m}}$ on each trial.

potentials most commonly arise from brief depolarizations. The mean width and rise time of the STA calculated from visually evoked spikes was $21.7 \pm 9.1$ and $10.2 \pm 4.9 \mathrm{msec}(n=37)$, respectively. These values were significantly smaller $(p \ll 0.01$; paired $t$ test) for action potentials occurring spontaneously (mean width $=31.8 \pm 10.0 \mathrm{msec}$ ); mean rise time $=18.4 \pm 4.8 \mathrm{msec})$ (Fig. 9C,D). To control for the possibility that the STA width might simply be a function of a cell's firing rate, we computed the STA from the visual responses recorded on each trial and compared trial-by-trial variations in firing rate and STA width in a subset of cells $(n=30)$. None of the cells tested showed a significant correlation between these two variables. These data suggest that visually evoked spikes arise from synaptic inputs that are more tightly synchronized compared with those occurring spontaneously. Thus, evoked spike count may depend not only on the magnitude of membrane depolarization evoked by the stimulus, but also on the amplitude of the high-frequency components in the $V_{\mathrm{m}}$ fluctuations.

We tested this notion by comparing the linear correlations between each cell's evoked spike count, the integrated power of the evoked $V_{\mathrm{m}}$ in the range of $20-70 \mathrm{~Hz}$ ( $\gamma$-band power), and the mean evoked $V_{\mathrm{m}}$. These calculations were performed across all trials on a $1 \mathrm{sec}$ epoch of activity immediately after the onset of the visual response (Fig. 10). To test for interactions among these parameters, we also computed the multiple regression between spike count, $\gamma$-band power, and the mean $V_{\mathrm{m}}$. The results are given in Table 1. Interestingly, variations in evoked spike count were most strongly correlated with variations in the amplitude of the $\gamma$-band fluctuations of $V_{\mathrm{m}}$. Thirty-nine of 52 cells examined (75\%) displayed a significant correlation. Even for those cells in which the correlation was statistically insignificant, the STA indicated that spikes nevertheless arose from brief depolarizing transients. Conversely, and perhaps counterintuitively, only $42 \%$ of the cells showed a significant correlation between spike count and mean evoked $V_{\mathrm{m}}$. A similar value (37\%) was obtained for the correlation of $\gamma$-band power and the mean $V_{\mathrm{m}}$. The multiple regression analysis revealed a slight increase in the strength of the correlation, indicating that spike count depended on both the mean $V_{\mathrm{m}}$ and the $\gamma$-band power.

\section{Variation in action potential threshold}

The relation between spike count and high-frequency fluctuations of $V_{\mathrm{m}}$ suggested to us that action potential threshold might vary over a relatively broad range of $V_{\mathrm{m}}$. We reasoned that spike threshold should depend on the rate of change of $V_{\mathrm{m}}$ before a spike and that variations in the temporal structure of the $V_{\mathrm{m}}$ fluctuations would give rise to variations in threshold (Azouz and Gray, 1998). To explore this possibility, we computed $V_{\mathrm{m}}$ at spike threshold for each visually evoked action potential and compared this distribution with all the subthreshold $V_{\mathrm{m}}$ values during the visual responses. We discovered that all the cells in our sample displayed relatively large variations in action potential threshold. Figure 11 shows examples of the raw data collected from two cells, one with a low variation $(\mathrm{SD}=1.8 \mathrm{mV})$ and the other with a larger variation in spike threshold $(\mathrm{SD}=2.7 \mathrm{mV})$. The distributions of evoked $V_{\mathrm{m}}$ and spike threshold are shown for the same two cells in Figure 12. It is apparent from these examples that spike threshold can vary substantially and that there are many epochs when $V_{\mathrm{m}}$ is more depolarized than the minimum threshold, although the cells fail to generate a spike. To quantify the range of spike threshold variation in our sample of cells, we computed the grand mean of the SD of spike threshold. This value $(2.3 \pm 1.5 \mathrm{mV})$ indicates that on average the spike threshold for any given cell will vary over a range of $\sim 9.2 \mathrm{mV}$ (i.e., $\pm 2 \sigma$ ). 
A

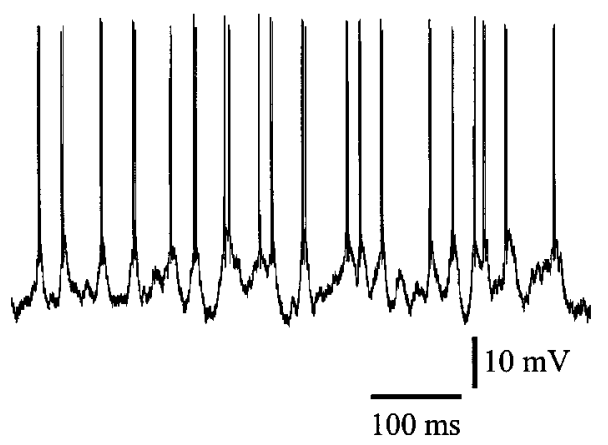

$\mathrm{C}$

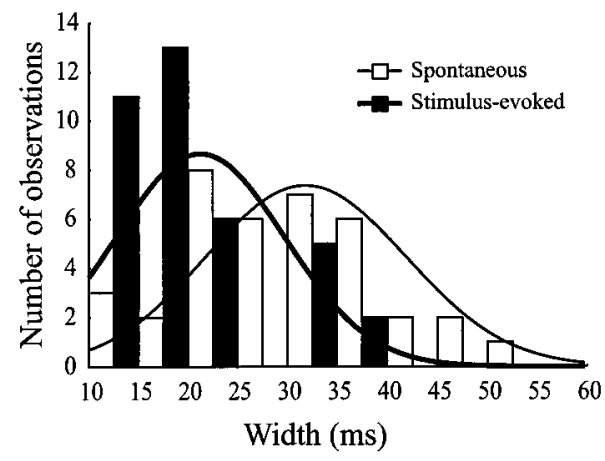

B

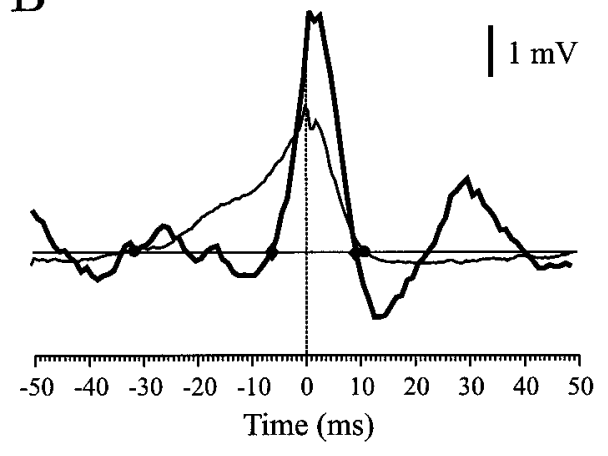

D

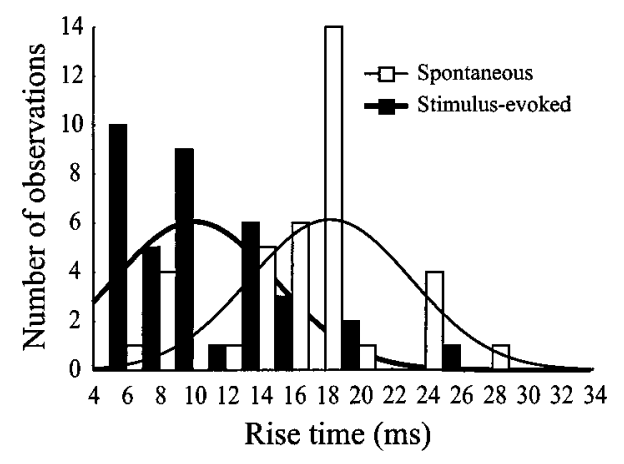

Figure 9. STA of $V_{\mathrm{m}}$ reveals the time course of depolarization leading to action potentials. $A$, This plot illustrates an epoch of raw data sampled during the response to a visual stimulus in a chattering cell. $B$, STA of $V_{\mathrm{m}}$ for all the action potentials recorded on 20 consecutive trials during spontaneous (thin line, 232 spikes) and stimulus-evoked (thick line, 1214 spikes) activity. The filled circles (spontaneous) and filled diamonds (evoked) mark the points at which the mean voltage crosses a predefined baseline. The time elapsed between these points is defined as the width of the peak in the STA, and the time between the leading point and time point 0 is defined as the rise time of the peak in the STA. $C, D$, These plots show the distribution of peak widths $(C)$ and rise times $(D)$ and associated Gaussian fits for the spontaneous and visually evoked data across the entire sample of cells $(n=37)$.

\section{Dependence of response strength on spontaneous- evoked $\boldsymbol{V}_{\mathrm{m}}$ correlation}

Our finding of a close correlation between spontaneous and evoked $V_{\mathrm{m}}$ suggested that this relationship might be influenced by the strength of cellular responses. To test this notion, we repeated two of the correlation measurements on data collected from a subset of cells $(n=13)$ that were presented with a range of spatial frequencies. This enabled us to determine whether the correlations were in any way related to the response strength of the cells. Each of the cells was presented with four to five different spatial frequencies. The mean firing rates were calculated and expressed as a percentage of the maximum response $(100 \%)$. For each cell, we then computed the spontaneous $V_{\mathrm{m}}$ to evoked $V_{\mathrm{m}}$ correlation and the spontaneous $V_{\mathrm{m}}$ to evoked spike-count correlation as a function of normalized firing rate. The resulting four to five data points were evaluated to determine whether the correlation strength or slope was related to the cell's firing rate. The results of these calculations are shown for one of the cells in Figure 13. In this example, the strength, but not the slope, of the spontaneous $V_{\mathrm{m}}$ to evoked $V_{\mathrm{m}}$ correlation was linearly related to the response strength of the cell (Fig. 13C). This demonstrates that spontaneous fluctuations in $V_{\mathrm{m}}$ are more tightly correlated to the evoked $V_{\mathrm{m}}$ when the cell is responding to an optimal stimulus and therefore exhibiting a more vigorous response. However, this relation does not translate into a similar correlation between spontaneous $V_{\mathrm{m}}$ to evoked spike count. Neither the magnitude of the correlation nor the regression slope was related to the response strength of the cell (Fig. 13D).

Although this example does provide evidence for a correlation between spontaneous and evoked activity as a function of response strength, the overall pattern was weaker. Five of 13 cells $(36 \%)$ displayed a significant correlation between response magnitude and $V_{\mathrm{m}}$ correlation strength, and four cells (29\%) showed a similar relation between response magnitude and $V_{\mathrm{m}}$ correlation slope. Only two (14\%) and three cells (21\%), respectively, showed a significant correlation between response magnitude and spontaneous $V_{\mathrm{m}}$ to evoked spike-count correlation strength and regression slope. Thus, response strength appears to influence the relation between spontaneous and evoked activity, but under the conditions of our experiments this influence is relatively weak.

\section{Network events underlying spontaneous fluctuations}

It has been demonstrated previously that spontaneous fluctuations in cortical activity are highly structured events in the spatial and temporal domain (Nelson et al., 1992; Arieli et al., 1995, 1996). When one cell in the cortical network fires spontaneously, many other nearby as well as distant cells fire at roughly the same time. This loose synchronization of spontaneous activity is thought to give rise to the comparatively large fluctuations of $V_{\mathrm{m}}$ that we have observed here. To test this simple prediction, we performed simultaneous recordings of intracellular $V_{\mathrm{m}}$ and extracellular spike activity from cells located within 100-500 $\mu \mathrm{m}$ $(n=40)$. In 33 of these paired recordings $(82.5 \%)$, we found that spontaneous discharges of the extracellular units were correlated with depolarizations of the intracellularly recorded $V_{\mathrm{m}}$. An example of this result is shown in Figure 14. These data indicate that the large fluctuations in $V_{\mathrm{m}}$ occurring spontaneously are likely to result from synchronous synaptic input converging onto cells.

\section{DISCUSSION}

Membrane potential correlations, intrinsic membrane properties, and network dynamics

Our results demonstrate a simple but important principle of neuronal function in the cerebral cortex. The $V_{\mathrm{m}}$ depolarization evoked by a stimulus is proportional to the $V_{\mathrm{m}}$ of the cell when the 
A
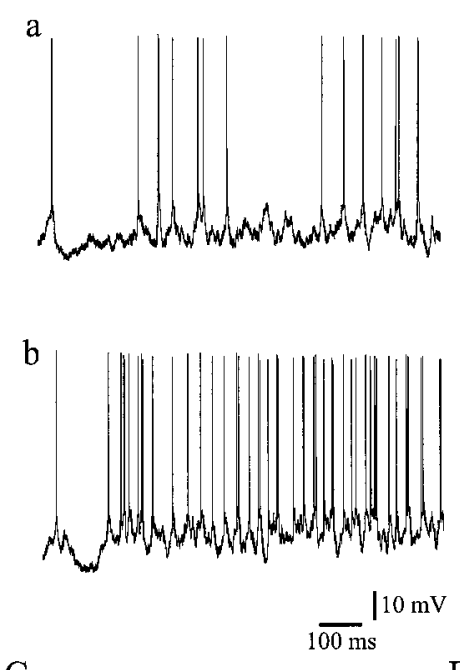

C

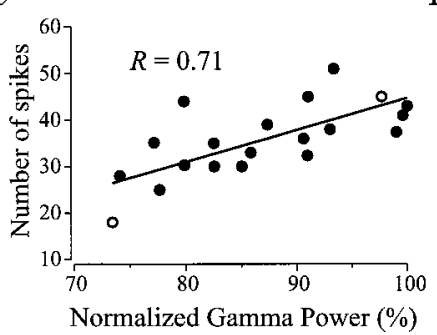

B

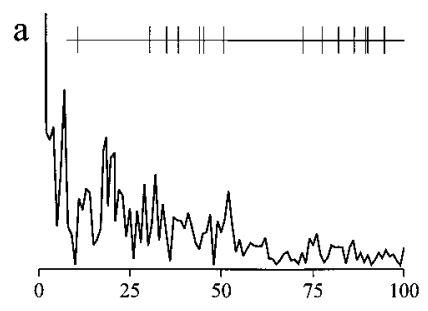

b

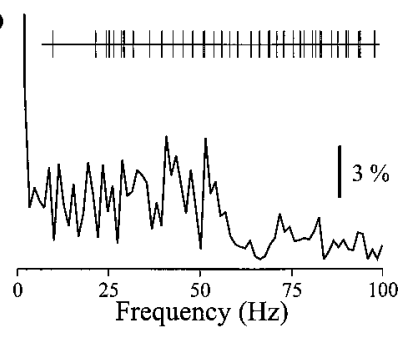

D

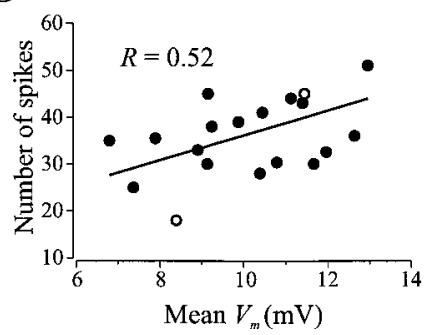

Figure 10. The number of visually evoked spikes is linearly correlated with the $\gamma$-band power in the $V_{\mathrm{m}}$ and more weakly correlated to the mean $V_{\mathrm{m}} \cdot A$, Raw data collected on two separate trials $(a, b)$ from the same cell. $B$, Fourier power spectra of the corresponding median-filtered $V_{\mathrm{m}}$ traces in $A$, and the extracted spike train (top traces). $C$, Scatter plot of the number of spikes occurring in the first $1024 \mathrm{msec}$ of the response versus the normalized $\gamma$ power $(20-70 \mathrm{~Hz})$ in the median-filtered $V_{\mathrm{m}} . D$, Scatter plot of the number of spikes versus the mean evoked response $V_{\mathrm{m}}$ for the same $1024 \mathrm{msec}$ epoch of data. The calibration bar in $B$ represents the percentage of power with respect to the peak value at the DC level. In these examples, firing rate is enhanced when both the $\gamma$-band power and the mean $V_{\mathrm{m}}$ are enhanced.

stimulus is presented. A similar relation also holds for spontaneous fluctuations of $V_{\mathrm{m}}$. These correlations in $V_{\mathrm{m}}$ extend for several hundred milliseconds, and their decay over time is attributable in part to a decrease in the percentage of cells that maintain the correlation. However, partial correlation and autocorrelation analyses revealed that the long-lasting correlations of $V_{\mathrm{m}}$ are attributable to the high correlation that occurs between adjacent epochs of activity and are thus not the result of lowfrequency fluctuations in $V_{\mathrm{m}}$. Although qualitatively similar, our results differ from those reported by Arieli et al. (1996). We found that the decay time constant of the $V_{\mathrm{m}}$ autocorrelation was 40 msec on average, whereas they reported a decay rate of $\sim 100$ msec for the optical signals produced by voltage-sensitive dyes. This difference is likely to stem from the obvious differences in origin between the two types of signals. Intracellular $V_{\mathrm{m}}$ largely reflects the synaptic input to a cell from a limited part of the network, whereas the surface recorded optical signal reflects the activity of a very large population of neurons.

It is perhaps somewhat surprising, however, that the relationship we and Arieli et al. (1996) have observed is so clearly linear. Given the variety of voltage-gated conductances possessed by cortical neurons, and the complexity of their somatic and den-

dritic distributions (Johnston et al., 1996; Yuste and Tank, 1996), one might expect to observe various nonlinear correlations. Consistent with this reasoning, we found that the strength of correlation increased when the cells were hyperpolarized, indicating that voltage-gated conductances contribute to the variability of sensory responses. Despite this effect, the vast majority of cells maintained a strong linear correlation under control conditions in which voltage-gated conductances are activated. This suggests that cortical neurons possess mechanisms to linearize their responses to synaptic input (Jagadeesh et al., 1993). Evidence both for and against this notion has come from recent in vitro studies. Using cultured hippocampal neurons, Cash and Yuste (1998) demonstrated that a balance between NMDA and $I_{\mathrm{A}}$ conductances maintained linear summation of the responses to depolarizing inputs independent of dendritic location. In contrast, Margulis and Tang (1998) demonstrated that dendritically located voltage-gated sodium conductances can supralinearly boost the temporal summation between spatially and temporally coincident synaptic inputs. These findings indicate that nonlinear mechanisms clearly play a role in synaptic integration, but it is conceivable that they may act in a balanced manner to maintain the linearity of summation to synaptic input (Cash and Yuste, 1998).

With respect to $V_{\mathrm{m}}$ fluctuations, our observations suggest that response variability arises in large part from the spontaneous synaptic activity in the cortical network (Arieli et al., 1995, 1996; Pare et al., 1997, 1998; Zador, 1998). This interpretation is compatible with the theoretical framework put forth by Aertsen and colleagues (Aertsen et al., 1989; Boven and Aertsen, 1990; Aertsen and Preissl, 1991; Vaadia and Aertsen, 1992). These authors have argued that the effective connectivity between neurons depends on the activity of the network in which they are embedded. Using multiple neuron recordings in behaving animals, they have demonstrated that correlated firing of cortical neurons is modulated on a fast time scale (Aertsen et al., 1989; Vaadia and Aertsen, 1992). Computer simulations of small networks of neurons demonstrate that such dynamic changes in correlation strength are directly related to the overall activity in the network (Boven and Aetsen, 1990; Aertsen and Preissl, 1991). Thus, the functional strength of synaptic connections in the network (i.e., the probability that firing in one cell will lead to firing in another cell to which it is synaptically connected) is activity dependent. The interpretation of this effect is relatively simple and can be paraphrased from Aertsen and Preissl (1991): "The network activity provides a background level, which, depending on its magnitude, will make the influence of one neuron on another more or less viable in eliciting activity. This effect of the network can be described as a control mechanism determining the operating point of neurons and thereby the efficacy of otherwise subthreshold connections." Thus, increased activity in the network leading to depolarization of a cell also increases the effective connectivity between this cell and those cells to which it is synaptically connected. If a visual stimulus is presented at the moment of enhanced activity, the response of a cell will be greater than if the network were relatively quiet. Aertsen and Preissel (1991) also demonstrated that the dynamic range of effective connectivity is inversely related to the coupling strength among cells in the network. Because the coupling strength among cortical neurons is low, this activity-dependent mechanism may provide a basis for the continuous linear relationships we have observed.

These interpretations are consistent with several aspects of our results. We found that the magnitude of $V_{\mathrm{m}}$ fluctuations is rela- 


\begin{tabular}{|c|c|c|c|}
\hline$\gamma$ power versus spike count & $\gamma$ power versus mean $V_{\mathrm{m}}$ & Mean $V_{\mathrm{m}}$ versus spike count & $\gamma$ power versus spike count versus mean $V_{\mathrm{m}}$ \\
\hline $0.68 \pm 0.15$ & $0.72 \pm 0.17$ & $0.69 \pm 0.19$ & $0.76 \pm 0.14$ \\
\hline$n=39 / 52(75 \%)$ & $n=19 / 52(37 \%)$ & $n=22 / 52(42 \%)$ & $n=39 / 52(75 \%)$ \\
\hline
\end{tabular}

A
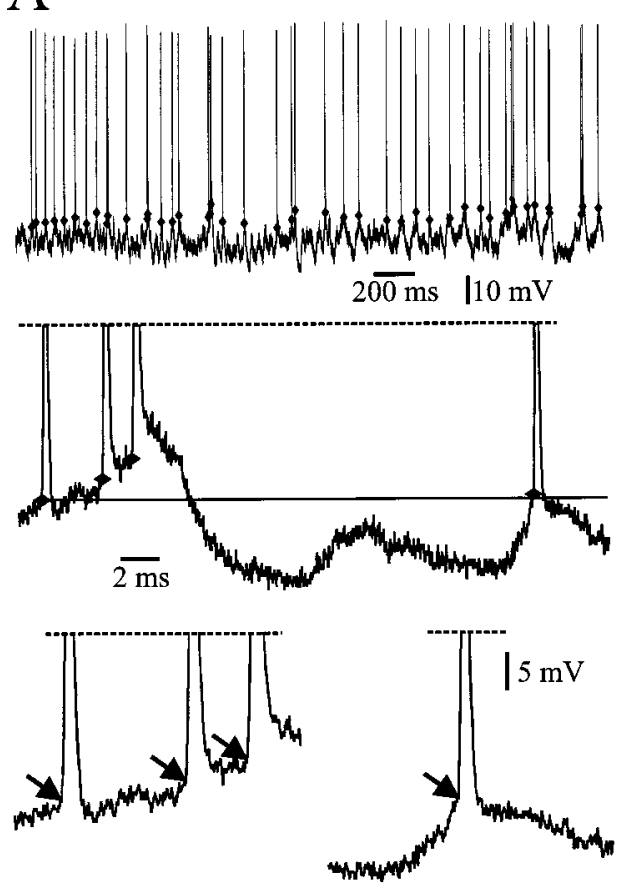
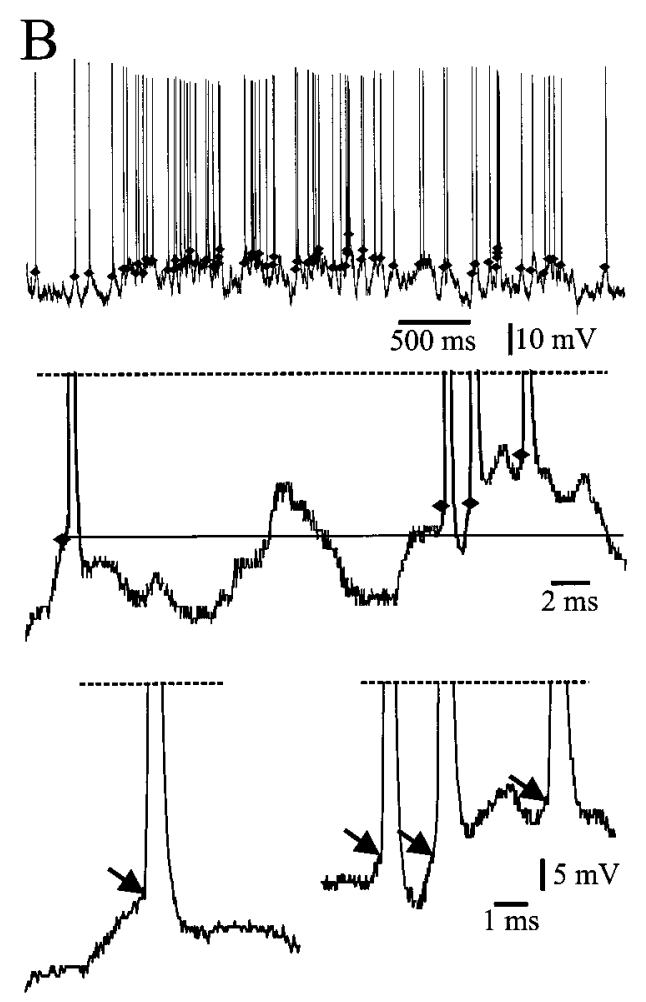

Figure 11. Visual cortical neurons exhibit dynamic variations in the threshold of spike initiation. The plots in $A$ and $B$ show examples of the raw data collected from two cells that exhibit different ranges of variation in action potential threshold. The top plots show the data collected during the visual response on a single trial for each cell. The middle and bottom plots display brief epochs of this data at medium and fast time scales, respectively. Action potential threshold is marked by a filled diamond in the top and middle plots and by arrows in the bottom plots. The continuous horizontal lines in the middle plots are used as a reference for comparison of different spike thresholds. The dashed lines indicate truncated spikes. tively large and typically spans a range of 15 to $20 \mathrm{mV}$. If one assumes that EPSP amplitudes are typically in the range of a few hundred microvolts (Thomson et al., 1993; Stevens and Zador, 1998) and excitatory synapses have relatively low release probabilities (Thomson and West, 1993; Stratford et al., 1996), then the large fluctuations of $V_{\mathrm{m}}$ in vivo are the result of hundreds of synaptic inputs impinging on a cell. Our finding that $V_{\mathrm{m}}$ fluctuations are correlated with spike discharges recorded extracellularly from nearby cells supports this interpretation. Further support comes from several other lines of evidence. Several groups have reported correlations in spontaneous discharge from neurons recorded simultaneously in the same or different cortical areas, between the cortex and thalamus, as well as in the two cerebral hemispheres (Nelson et al., 1992; Contreras and Steriade, 1995; Nowak et al., 1995). These findings and the theoretical predictions of Aertsen and colleagues (Aertsen et al., 1989; Boven and Aertsen, 1990; Aertsen and Preissl, 1991; Vaadia and Aertsen, 1992) are consistent with the results of Arieli et al. $(1995,1996)$ demonstrating that spontaneous cortical activity is highly structured both spatially and temporally. We conclude from these numerous observations that spontaneous cortical activity is not neuronal noise, which can be averaged away by some pooling process. Rather it represents a spatially and temporally coherent state of the cortical network that continuously fluctuates and plays a crucial role in regulating the response strength of cortical neurons to sensory inputs.

\section{Contribution of spike generation to response variability}

Another major finding of this study is that the fluctuations in response strength, measured by cellular firing rates and latencies, are greater than expected from the fluctuations in $V_{\mathrm{m}}$ alone. Visually evoked neuronal firing rates and spike latencies were only weakly correlated with spontaneous or visually evoked fluctuations in the mean $V_{\mathrm{m}}$, a result that held for brief $(128 \mathrm{msec})$ as well as longer $(1 \mathrm{sec})$ episodes of activity. Our finding that spike threshold can vary over a range of $\sim 9 \mathrm{mV}$ suggests that spike generation contributes to response variability. This effect could result from an intrinsic source of variance added by the spikegenerating mechanism. However, in agreement with other investigators (Mainen and Sejnowski, 1995; Carandini et al., 1996; Nowak et al., 1997a; Stevens and Zador, 1998), we argue that the increased variance of spike output is consistent with a reliable spike-generating mechanism endowed with specific voltage- and time-dependent properties. Several of our findings support this conclusion. Calculation of the spike-triggered average of $V_{\mathrm{m}}$ revealed that spikes arise most often from transient fluctuations of $V_{\mathrm{m}}$ having a mean rise time near $10 \mathrm{msec}$ (Nowak et al., 1997a,b). Similarly, we found that visually evoked neuronal firing rates are closely correlated with the amplitude of $V_{\mathrm{m}}$ fluctuations in the $\gamma$ frequency band $(20-70 \mathrm{~Hz})$. These data indicate that given a sufficiently strong depolarizing input, shaped by the properties of the visual stimulus, the spike-generating mechanism acts as a high-pass filter (Mainen and Sejnowski, 1995; Carandini 


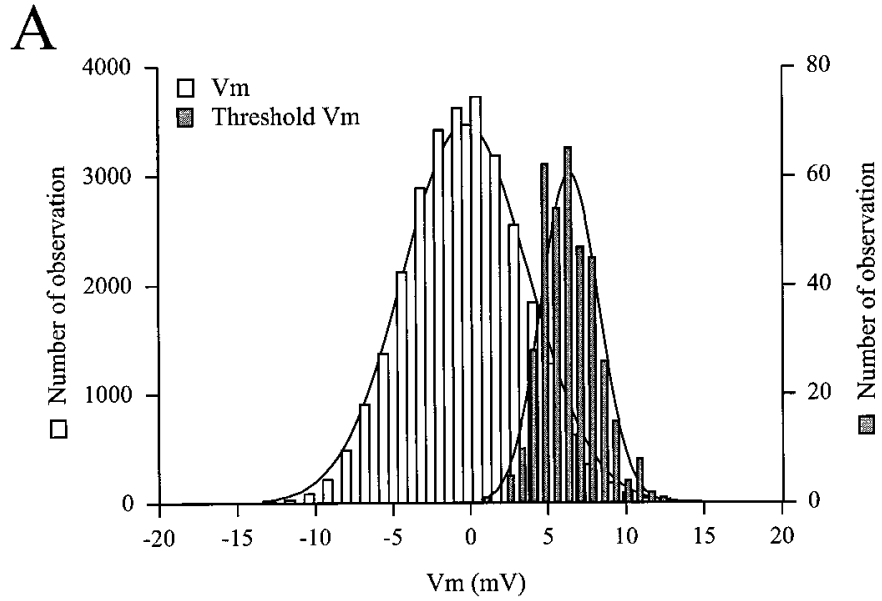

B

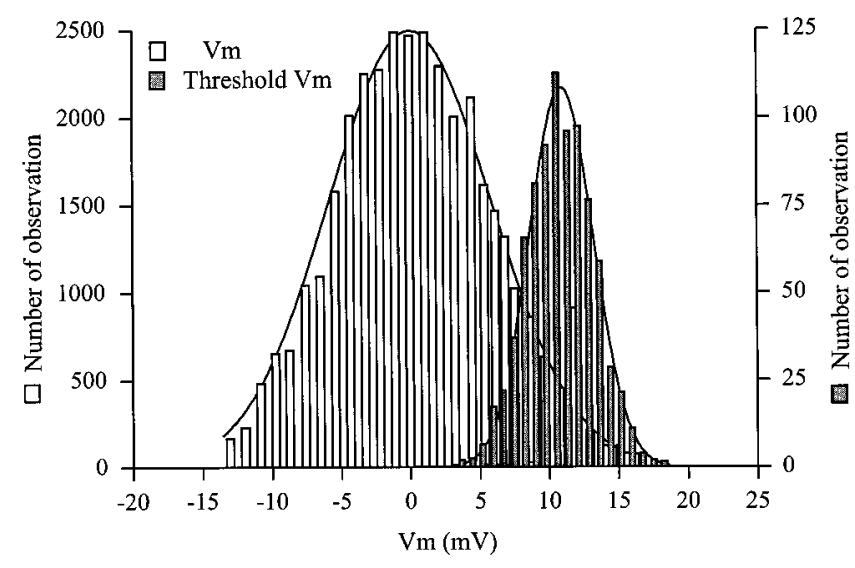

Figure 12. $A, B$, Distributions of $V_{\mathrm{m}}$ (unfilled bars) and spike threshold ( filled bars) accumulated from the visual responses across all trials for the same two cells shown in Figure 11. The distributions of $V_{\mathrm{m}}$ were sampled during the periods when the cells were not firing an action potential. Note that the distributions overlap substantially, indicating that $V_{\mathrm{m}}$ can often exceed the minimum threshold without the cell firing a spike.

et al., 1996; Nowak et al., 1997a). Thus variance may be added to the spike train not because the spike-encoding mechanism is noisy, but because variations in the high-frequency components of $V_{\mathrm{m}}$ lead to variations in spike discharge.

Our finding that action potential threshold varies over a relatively wide range supports this conjecture. Given the voltage- and time-dependent properties of sodium channel activation and inactivation (Hodgkin and Huxley, 1952), action potential threshold should vary according to the rate of change of $V_{\mathrm{m}}$ preceding a spike. This has been observed in a number of studies (Calvin, 1974; Schlue et al., 1974; Schwindt and Crill, 1982; Stafstrom et al., 1984; Heck et al., 1993), and we have found it to be true for cortical neurons in vivo. In a separate study, we found that action potential threshold is correlated with the rate of change of $V_{\mathrm{m}}$ preceding a spike (Azouz and Gray, 1998). Spikes exhibited a low voltage threshold when preceded by rapid depolarization, and the threshold voltage increased as the rate of $V_{\mathrm{m}}$ depolarization preceding a spike decreased. These findings are consistent with the notion that the spike-generating mechanism acts as a highpass filter (Carandini et al., 1996) and can account for our finding that evoked spike count and latency is weakly correlated with fluctuations in the mean $V_{\mathrm{m}}$.
A

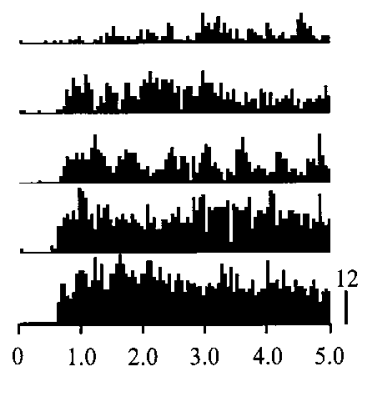

B

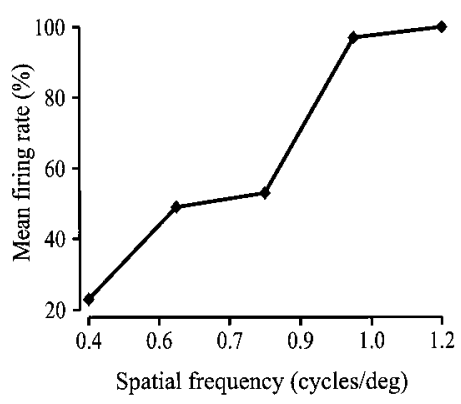

$\mathrm{C}$

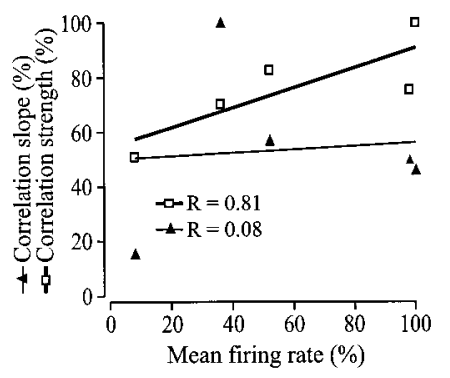

D

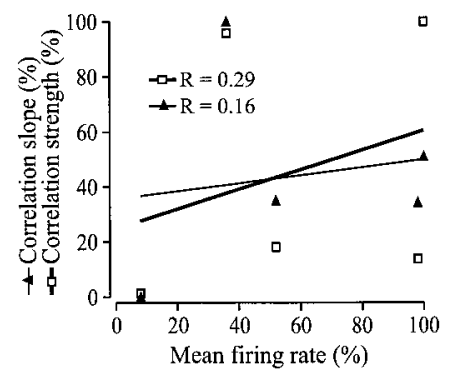

Figure 13. The correlation between spontaneous and evoked activity is dependent on response strength. $A$, Peristimulus time histograms of spike activity in response to five different spatial frequencies of a drifting square-wave grating $\left(0.4-1.2\right.$ cycles $/{ }^{\circ}$ in steps of 0.2 cycles $\left./{ }^{\circ}\right)$. The calibration bar at the bottom right indicates the number of spikes/bin. $B$, Normalized tuning curve for the cell computed from the mean firing rate during the first second of the visual response. $C$, Scatter plot of the normalized strength $(\square$, thick line $)$ and regression slope ( $\mathbf{\Lambda}$, thin line $)$ of the correlation between the spontaneous and evoked $V_{\mathrm{m}}$ and the normalized response strength to gratings of different spatial frequencies. $D$, Scatter plot of the normalized strength $(\square$, thick line $)$ and regression slope $(\boldsymbol{\Lambda}$, thin line $)$ of the correlation between the spontaneous $V_{\mathrm{m}}$ and evoked spike count and the normalized response strength to gratings of different spatial frequencies.

These conclusions, however, rest largely on the assumption that our measurements of action potential threshold reflect the voltage fluctuations occurring at the site of spike initiation. We generally assume, but cannot confirm, that the majority of our intracellular impalements occur at or near the soma. Several studies have reported that action potentials in cortical neurons are initiated in the axon initial segment or the first node of Ranvier (Gogan et al., 1983; Colbert and Johnston, 1996; Stuart et al., 1997). Thus, our measurements of action potential threshold could deviate from the actual threshold at the site of initiation if these compartments are not isopotential. Under these conditions, fluctuations of $V_{\mathrm{m}}$ in the soma could obscure the threshold behavior at the site of initiation. We cannot exclude the possibility that this effect contributes to our measure of variation in spike threshold. However, Gogan et al. (1983) demonstrated that the electronic length of the axon initial segment of spinal motoneurons is relatively short. If this is also true for cortical neurons, as suggested by the study of Colbert and Johnston (1996), the voltage fluctuations in the first node of Ranvier should be similar to those observed in the soma. Our finding that spike threshold is correlated with the rate of change of $V_{\mathrm{m}}$ is consistent with this interpretation (Azouz and Gray, 1998). 


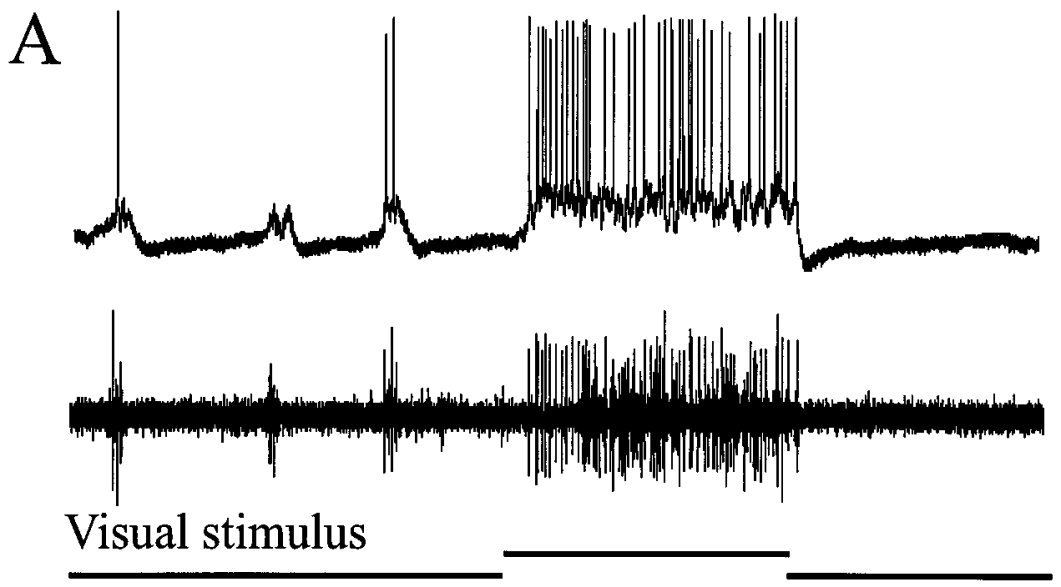

B

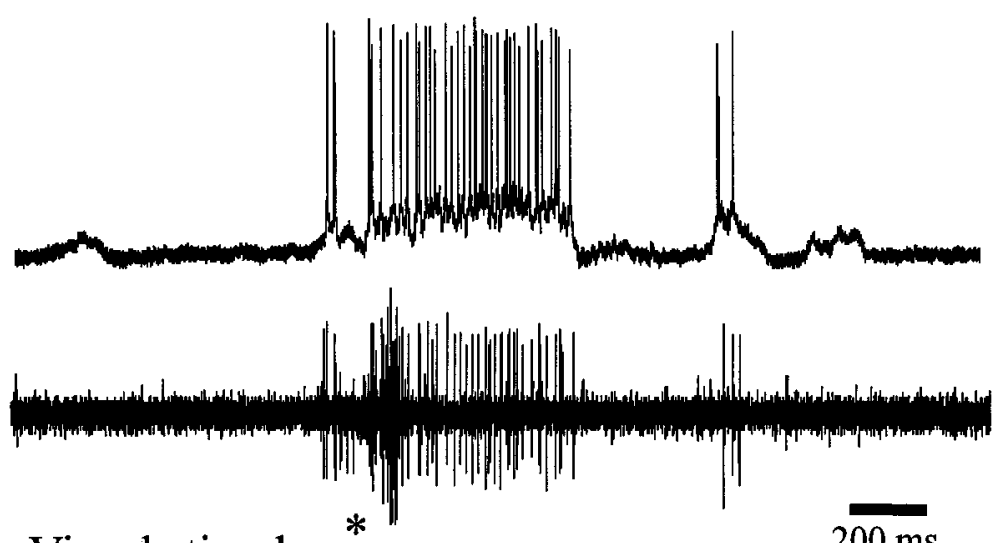

Visual stimulus

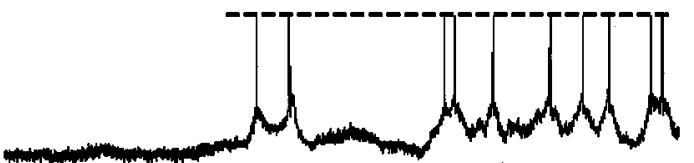

I $10 \mathrm{mV}$

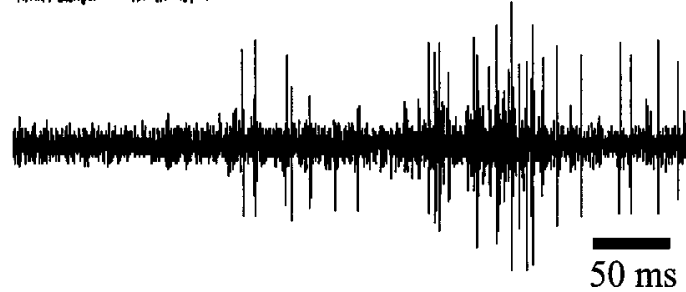

$200 \mu \mathrm{V}$
Figure 14. Extracellular spike activity is correlated with fluctuations in $V_{\mathrm{m}}$. Two examples of simultaneously recorded extracellular and intracellular activity taken from the same electrode penetrations. The recording electrodes were $\sim 500 \mu \mathrm{m}$ apart. $A$, The top trace shows the $V_{\mathrm{m}}$ of an intracellularly impaled cell during spontaneous and evoked activity. The bottom trace shows the extracellular multiunit activity recorded at the same time. $B$, The same extracellular units as in $A$, but a different intracellularly impaled cell. The epoch marked with an asterisk is shown at an expanded time scale in the bottom panels. Note that the spontaneous spike activity of the extracellularly recorded units is correlated with brief periods of depolarization in the intracellularly recorded units.

\section{Relation between response synchronization, variability, and temporal precision}

Our findings that firing rate and threshold are linked to highfrequency fluctuations in $V_{\mathrm{m}}$ supports the argument, put forth elsewhere (Nowak et al., 1997a,b; Reich et al., 1997; Stevens and Zador, 1998; Zador, 1998), that the contrasting behaviors of response variability and high temporal precision are related to the incidence of synchronous synaptic input onto cortical neurons. The high-pass characteristics of the spike-generating mechanism make it sensitive to transient fluctuations in $V_{\mathrm{m}}$ that are likely to be generated by synchronous inputs (Jagadeesh et al., 1992; Nowak et al., 1997b; Stevens and Zador, 1998). This argues that cortical neurons can act as detectors of coincident synaptic inputs (Abeles, 1982; Konig et al., 1996; Azouz and Gray, 1998). Thus, variations in high-frequency synaptic input onto a cortical neuron will lead to corresponding variations in the cell's firing rate and the temporal pattern of its spike train. Therefore, response variability and the irregular patterns of interspike intervals observed in cortical neurons represent two sides of the same coin, both of which can be explained by a spike-encoding mechanism that is sensitive to variations in high-frequency synaptic input. This argues that high-frequency synaptic input plays at least two important roles in cortical function. It can extend the temporal bandwidth of cortical neurons and thereby increase the transmission of visually evoked information (Carandini et al., 1996). Complementary to this, the greater sensitivity to high-frequency fluctuations could serve to convey precise information about a time-varying sensory stimulus (Bair and Koch, 1996; Buracas et al., 1998). We speculate that the cortex possesses an intrinsic mechanism to generate high-frequency fluctuations in the form of 
synchronous $\gamma$-band activity (Singer and Gray, 1995). This activity may arise in part from a distinct class of cortical neuron, called chattering cells, that are capable of generating $\gamma$-band activity by virtue of their intrinsic membrane properties (Gray and McCormick, 1996).

It must be pointed out, however, that part of our results also provide support for an alternative hypothesis. It has been argued elsewhere that sensory processing is most efficient when neuronal activity is decorrelated so that it conveys independent messages in a nonredundant manner (Barlow, 1961). Our finding that the correlation strength observed in the $V_{\mathrm{m}}$ fluctuations increases when the cells are hyperpolarized indicates that the intrinsic conductances of cortical neurons contribute to an increase in response variability and a possible decrease in response covariance. This effect may represent an intrinsic cellular mechanism, one that is conceivably controlled by neuromodulatory systems to maintain a decorrelation among the spike discharges of cortical neurons.

\section{REFERENCES}

Abeles M (1982) Role of the cortical neuron: integrator or coincidence detector? Isr J Med Sci 18:83-92.

Abeles M, Bergman H, Margalit E, Vaadia E (1993) Spatiotemporal firing patterns in the frontal cortex of behaving monkeys. J Neurophysiol 70:1629-1638.

Aertsen A, Preissel H (1991) Dynamics of activity and connectivity in physiological neuronal networks. In: Nonlinear dynamics and neuronal networks (Schuster HG, ed), pp 281-301. Weinheim, Germany: VCH Verlag.

Aertsen A, Gerstein GL, Habib MK, Palm G (1989) Dynamics of neuronal firing correlation: modulation of "effective connectivity." J Neurophysiol 61:900-917.

Aertsen AM, Smolders JW, Johannesma PI (1979) Neural representation of the acoustic biotope: on the existence of stimulus-event relations for sensory neurons. Biol Cybern 32:175-185.

Arieli A, Shoham D, Hildesheim R, Grinvald A (1995) Coherent spatiotemporal patterns of ongoing activity revealed by real-time optical imaging coupled with single-unit recording in the cat visual cortex. J Neurophysiol 73:2072-2093.

Arieli A, Sterkin A, Grinvald A, Aertsen A (1996) Dynamics of ongoing activity: explanation of the large variability in evoked cortical responses. Science 273:1868-1871.

Azouz R, Gray CM (1998) Spike generation in cortical neurons in vivo: dynamic variability of action potential threshold. Soc Neurosci Abstr 24:497.4.

Bach M, Krueger J (1986) Correlated neuronal variability in monkey visual cortex revealed by a multi-microelectrode. Exp Brain Res 61:451-456.

Bair W, Koch C (1996) Temporal precision of spike trains in extrastriate cortex of the behaving macaque monkey. Neural Comp 8:1185-1202.

Barlow HB (1961) Possible principles underlying the transformation of sensory messages. In: Sensory communication (Rosenblith WA, ed), pp 217-234. Cambridge, MA: MIT.

Bernander O, Douglas RJ, Martin KA, Koch C (1991) Synaptic background activity influences spatiotemporal integration in single pyramidal cells. Proc Natl Acad Sci USA 88:11569-11573.

Boven KH, Aertsen A (1990) Dynamics of activity in neuronal networks give rise to fast modulations of functional connectivity. In: Parallel processing in neural systems and computers (Eckmiller R, Hartmann G, Hauske G, eds), pp 53-56. Amsterdam: Elsevier.

Britten KH, Shadlen MN, Newsome WT, Movshon JA (1992) The analysis of visual motion: a comparison of neuronal and psychophysical performance. J Neurosci 12:4745-4765.

Bullock TH (1970) The reliability of neurons. J Gen Physiol 55:565-584.

Buracas GT, Zador AM, DeWeese MR, Albright TD (1998) Efficient discrimination of temporal patterns by motion sensitive neurons in primate visual cortex. Neuron 20:959-969.

Burns BD (1968) The uncertain nervous system. London: Edward Arnold Ltd.

Calvin WH (1974) Three modes of repetitive firing and the role of threshold time course between spikes. Brain Res 59:341-346.
Calvin WH, Stevens CF (1968) Synaptic noise and other sources of randomness in motoneuron interspike intervals. J Neurophysiol 31:574-587.

Carandini M, Mechler F, Leonard CS, Movshon A (1996) Spike train encoding by regular-spiking cells of the visual cortex. J Neurophysiol 76:3425-3441.

Cash S, Yuste R (1998) Input summation by cultured pyramidal neurons is linear and position-independent. J Neurosci 18:10-15.

Colbert CM, Johnston D (1996) Axonal action-potential initiation and $\mathrm{Na}+$ channel densities in the soma and axon initial segment of subicular pyramidal neurons. J Neurosci 16:6676-6686.

Connors BW, Gutnick MJ (1990) Intrinsic firing patterns of diverse neocortical neurons. Trends Neurosci 13:99-104.

Contreras D, Steriade M (1995) Cellular basis of EEG slow rhythms: a study of dynamic corticothalamic relationships. J Neurosci 15:604-622.

Dayhoff JE, Gerstein GL (1983) Favored patterns in spike trains. I. Detection. J Neurophysiol 49:1334-1348.

Dean AF (1981) The variability of discharge of simple cells in the cat striate cortex. Exp Brain Res 44:437-440.

de Ruyter van Steveninck RR, Lewen GD, Strong SP, Koberle R, Bialek W (1997) Reproducibility and variability in neural spike trains. Science 275:1805-1808.

Frostig RD, Frostig Z, Harper RM (1990) Recurring discharge patterns in multiple spike trains. I. Detection. Biol Cybern 62:487-493.

Gogan P, Gueritaud JP, Tyc-Dumont S (1983) Comparison of antidromic and orthodromic action potential of identified motor axons in the cat's brain. J Physiol (Lond) 335:205-220.

Gray CM, McCormick DA (1996) Chattering cells: superficial pyramidal neurons contributing to the generation of synchronous oscillations in visual cortex. Science 274:109-113.

Gray CM, Azouz R (1997) The influence of background activity on visual response properties of cortical cells in the cat's striate cortex. Soc Neurosci Abstr 23:13-11.

Gray CM, Engel AK, Koenig P, Singer W (1992) Synchronization of oscillatory neuronal responses in cat striate cortex: temporal properties. Vis Neurosci 8:337-347.

Gray CM, Maldonado P, Wilson M, McNaughton B (1995) Tetrodes markedly improve the reliability and yield of multiple single unit isolation from multiunit recordings in cat striate cortex. J Neurosci Methods 63:43-54.

Gur M, Beylin A, Snodderly DM (1997) Response variability of neurons in primary visual cortex (V1) of alert monkeys. J Neurosci 17:2914-2920.

Heck D, Rotter S, Aertsen A (1993) Spike generation in cortical neurons: probabilistic threshold function shows intrinsic and long-lasting dynamics. In: Brain theory (Aertsen A, ed), pp 241-249. Amsterdam: Elsevier.

Heggelund P, Albus K (1978) Response variability and orientation discrimination of single cells in striate cortex of cat. Exp Brain Res 32:197-211.

Henry GH, Bishop PO, Tupper RM, Dreher B (1973) Orientation specificity and response variability of cells in the striate cortex. Vision Res 13:1771-1779.

Hodgkin AL, Huxley AF (1952) A quantitative description of membrane current and its application to conduction and excitation in nerve. J Physiol (Lond) 117:500-544.

Holt GR, Softky WR, Koch C, Douglas RJ (1996) A comparison of discharge variability in vitro and in vivo in cat visual cortex neurons. J Neurophysiol 75:1806-1814.

Humphrey AL, Saul AB (1992) Action of brain stem reticular afferents on lagged and nonlagged cells in the cat lateral geniculate nucleus. J Neurophysiol 68:673-691.

Jagadeesh B, Gray CM, Ferster D (1992) Visually evoked oscillations of membrane potential in neurons of cat striate cortex studied with in vivo whole cell patch recording. Science 257:552-554.

Jagadeesh B, Wheat HS, Ferster D (1993) Linearity of summation of synaptic potentials underlying direction selectivity in simple cells of the cat visual cortex. Science 262:1901-1904.

Johnston D, Magee JC, Colbery CM, Christie BR (1996) Active properties of neuronal dendrites. Annu Rev Neurosci 19:165-186.

Kawaguchi Y, Kubota Y (1997) GABAergic cell subtypes and their synaptic connections in rat frontal cortex. Cereb Cortex 7:476-486.

Komatsu Y, Nakajima S, Toyama K, Fetz EE (1988) Intracortical connectivity revealed by spike-triggered averaging in slice preparations of cat visual cortex. Brain Res 442:359-362. 
Konig P, Engel AK, Singer W (1996) Integrator or coincidence detector? The role of the cortical neuron revisited. Trends Neurosci 19:130-137.

Larkman A, Mason A (1990) Correlations between morphology and electrophysiology of pyramidal neurons in slices of rat visual cortex. I. Establishment of cell classes. J Neurosci 10:1407-1414.

Lee D, Port NL, Kruse W, Georgopolous AP (1998) Variability and correlated noise in the discharge of neurons in motor and parietal areas of the primate cortex. J Neurosci 18:1161-1170.

Mainen ZF, Sejnowski TJ (1995) Reliability of spike timing in neocortical neurons. Science 268:1503-1506.

Margulis M, Tang C-M (1998) Temporal integration can readily switch between sublinear and supralinear summation. J Neurophysiol 79:2809-2813.

Mason A, Larkman A (1990) Correlations between morphology and electrophysiology of pyramidal neurons in slices of rat visual cortex. II. Electrophysiology. J Neurosci 10:1415-1428.

McCormick DA, Connors BW, Lighthall JW, Prince DA (1985) Comparative electrophysiology of pyramidal and sparsely spiny stellate neurons of the neocortex. J Neurophysiol 54:782-806.

Nelson JI, Salin PA, Munk MH, Arzi M, Bullier J (1992) Spatial and temporal coherence in cortico-cortical connections: a cross-correlation study in areas 17 and 18 in the cat. Vis Neurosci 9:21-37.

Nowak LG, Munk MH, James AC, Nelson JI, Bullier J (1995) Structural basis of cortical synchronization. I. Three types of interhemispheric coupling. J Neurophysiol 74:2379-2400.

Nowak LG, Sanchez-Vives MV, McCormick DA (1997a) Influence of low and high frequency inputs on spike timing in visual cortical neurons. Cereb Cortex 7:487-501.

Nowak LG, Sanchez-Vives MV, McCormick DA (1997b) Membrane potential trajectory preceding visually evoked action potentials in cat's visual cortex. Soc Neurosci Abstr 23:13-9.

Paradiso MA (1988) A theory for the use of visual orientation information which exploits the columnar structure of striate cortex. Biol Cybern 58:35-49.

Pare D, Lebel E, Lang EJ (1997) Differential impact of miniature synaptic potentials on the soma and dendrites of pyramidal neurons in vivo. J Neurophysiol 78:1735-1739.

Pare D, Shink E, Gaudreau H, Destexhe A, Lang EJ (1998) Impact of spontaneous synaptic activity on the resting properties of cat neocortical pyramidal neurons in vivo. J Neurophysiol 79:1450-1460.

Reich DS, Victor JD, Knight BW, Ozaki T, Kaplan E (1997) Response variability and timing precision of neuronal spike trains in vivo. J Neurophysiol 77:2836-2841.

Riehle A, Grun S, Diesmann M, Aertsen A (1997) Spike synchronization and rate modulation differentially involved in motor cortical function. Science 278:1950-1953.

Rose D (1979) An analysis of the variability of unit activity in the cat's visual cortex. Exp Brain Res 37:595-604.

Schiller PH, Finlay BL, Volman SF (1976) Short-term response variability of monkey striate neurons. Brain Res 105:347-349.

Schlue WR, Richter DW, Mauritz KH, Nacimiento AC (1974) Responses of cat spinal motoneuron somata and axons to linearly rising currents. J Neurophysiol 37:303-309.

Schwindt PC, Crill WE (1982) Factors influencing motoneurons rhythmic firing: results from a voltage-clamp study. J Neurophysiol 48:875-890.

Scobey RP, Gabor AJ (1989) Orientations discrimination sensitivity of single units in cat primary visual cortex. Exp Brain Res 77:398-406.

Shadlen MN, Newsome WT (1994) Noise, neural codes and cortical organization. Curr Opin Neurobiol 4:569-579.

Shadlen MN, Newsome WT (1998) The variable discharge of cortical neurons: implications for connectivity, computation and information coding. J Neurosci 18:3870-3896.
Shadlen MN, Britten KH, Newsome WT, Movshon JA (1996) A computational analysis of the relationship between neuronal and behavioral responses to visual motion. J Neurosci 16:1486-1510.

Singer W, Gray CM (1995) Visual feature integration and the temporal correlation hypothesis. Annu Rev Neurosci 18:555-586.

Snowden RJ, Treue S, Andersen RA (1992) The response of neurons in areas $\mathrm{V} 1$ and MT of the alert rhesus monkey to moving random dot patterns. Exp Brain Res 88:389-400.

Softky WR, Koch C (1993) The highly irregular firing of cortical cells is inconsistent with temporal integration of random EPSPs. J Neurosci 13:334-350.

Stafstrom CE, Schwindt PC, Flatman JA, Crill WE (1984) Properties of subthreshold response and action potential recorded in layer $\mathrm{V}$ neurons from cat sensorimotor cortex in vitro. J Neurophysiol 52:244-263.

Stevens CF, Zador AM (1998) Input synchrony and the irregular firing of cortical neurons. Nature Neurosci 1:210-217.

Stratford KJ, Tarczy-Hornoch K, Martin KAC, Bannister NJ, Jack JJB (1996) Excitatory synaptic inputs to spiny stellate cells in cat visual cortex. Nature 382:258-261.

Stuart G, Schiller J, Sakmann B (1997) Action potential initiation and propagation in rat neocortical pyramidal neurons. J Physiol (Lond) 505:617-632.

Thomson AM, West DC (1993) Fluctuations in pyramidal-pyramidal excitatory postsynaptic potentials modified by presynaptic firing pattern and postsynaptic membrane potential using paired intracellular recordings in rat neocortex. Neuroscience 54:329-346.

Thomson AM, Deuchars J, West DC (1993) Large, deep layer pyramidpyramid single axon EPSPs in slices of rat motor cortex display paired pulse and frequency-dependent depression, mediated presynaptically and self-facilitation, mediated postsynaptically. J Neurophysiol 70:2354-2369.

Tolhurst DJ, Movshon JA, Thompson ID (1981) The dependence of response amplitude and variance of cat visual cortical neurones on stimulus contrast. Exp Brain Res 41:414-419.

Tolhurst DJ, Movshon JA, Dean AF (1983) The statistical reliability of signals in single neurons in cat and monkey visual cortex. Vision Res 23:775-785.

Tomko GJ, Crapper DR (1974) Neuronal variability: nonstationary responses to identical visual stimuli. Brain Res 79:405-418.

Vaadia E, Aertsen A (1992) Coding and computation in the cortex: single neuron activity and cooperative phenomena. In: Information processing in the cortex (Aertsen A, Braitenburg V, eds), pp 81-121. New York: Springer.

van Kan PL, Scobey RP, Gabor AJ (1985) Response covariance in cat visual cortex. Exp Brain Res 60:559-563.

Villa AE, Abeles M (1990) Evidence for spatiotemporal firing patterns within the auditory thalamus of the cat. Brain Res 509:325-327.

Vogels R (1990) Population coding of stimulus orientation by striate cortical cells. Biol Cybern 64:25-31.

Vogels R, Orban GA (1990) How well do response changes of striate neurons signal differences in orientation: a study in the discriminating monkey. J Neurosci 10:3543-3558.

Vogels R, Spileers W, Orban GA (1989) The response variability of striate cortical neurons in the behaving monkey. Exp Brain Res 77:432-436.

Yuste R, Tank DW (1996) Dendritic integration in mammalian neurons, a century after Cajal. Neuron 16:701-716.

Zador A (1998) Impact of synaptic unreliability on the information transmitted by spiking neurons. J Neurophysiol 79:1219-1229.

Zohary E, Shadlen MN, Newsome WT (1994) Correlated neuronal discharge rate and its implication for psychophysical performance. Nature 370:140-143.

Zar JH (1996) Biostatistical analysis, Ed 3. Englewood Cliffs, NJ: Prentice Hall. 